\title{
Fuel-related Emissions from the Croatian Municipal Solid Waste Collection System in 2013: Mixed Municipal Waste
}

\author{
A. Grbeš, ${ }^{*}$ I. Ljubić, and Ž. Veinovića \\ a University of Zagreb, Faculty of Mining, Geology and Petroleum Engineering, Croatia \\ 'Humana Nova Zagreb, Croatia
}

\begin{abstract}
Waste removal (collection and landfilling) in the Republic of Croatia is the responsibility of the municipalities and local governments in 21 administrative units (counties). They entrust the respective economic activity to 208 private and public companies specialized in waste collection and treatment. Organised waste collection affects $99 \%$ of the population. The mixed waste from households and enterprises is at various frequencies collected at the door (kerbside collection) and transported by truck to a landfill, or processing plant.

This article aims to estimate fuel consumption and fuel-related airborne emissions from the collection of mixed municipal waste in Croatia in 2013. The input data and emission results are shown for Croatia and each Croatian county, in total, and relative to the number of inhabitants and mass of collected waste. Annual consumption of diesel for the collection of mixed waste is estimated at 10.6 million litres. At the county level, fuel consumption ranges from 87 thousand litres to 2.2 million litres, on average 504 thousand litres per county. Total emission of $\mathrm{CO}_{2}$ is estimated at $28000 \mathrm{t}$, which at county level ranges from 231 to $5711 \mathrm{t}$. Relative emission ranges from 3.3 to $13 \mathrm{~kg} \mathrm{CO}_{2}$ per capita (average $6.6 \mathrm{~kg}$ per capita), or $8.6-28.1 \mathrm{~kg} \mathrm{t}^{-1}$ of municipal waste (average $17 \mathrm{~kg} \mathrm{CO}_{2}$ per ton of municipal waste). The average values of $\mathrm{CO}_{2}$ emission from MSW collection that should also be the target values are 7-9 $\mathrm{kg}$ for mixed waste, and 8-15 $\mathrm{kg} \mathrm{CO}_{2}$ for separate waste streams. Apart from $\mathrm{CO}_{2}$ emission,

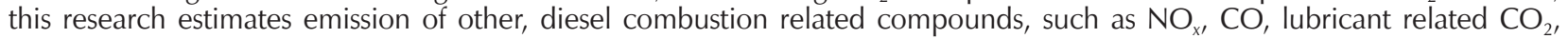
NMVOC, PM, f-BC, $\left.\mathrm{N}_{2} \mathrm{O}, \mathrm{SO}_{2}, \mathrm{NH}_{3}, \mathrm{~Pb}, \mathrm{ID}[1,2,3-\mathrm{cd}] \mathrm{P}, \mathrm{B}[\mathrm{k}] \mathrm{F}, \mathrm{B}[\mathrm{b}] \mathrm{F}, \mathrm{B}[\mathrm{a}] \mathrm{P}\right)$, as well as total distance of transport.
\end{abstract}

\section{Keywords}

Municipal solid waste, mixed waste stream, collection, fuel consumption, emissions

\section{Introduction}

This article aims to estimate emissions to air from the Croatian municipal solid waste (MSW) collection system. The estimate of fuel consumption is based on data delivered from half of the total number of Croatian companies in the business of MSW collection. ${ }^{1}$ The emissions to air are calculated using Tier 1 emission factors for heavy duty vehicles. Results are shown for each Croatian county, in total, and relative to the number of inhabitants and mass of collected waste. The time relevance of this research is for the year 2013, when the Croatian MSW recycling rate was only about $15 \%$. These data can be used as the reference level for landfilling/low recycling scenario in the studies on environmental efficiency of the various waste management scenarios.

The collection and transport of municipal solid waste is an important unit process of the waste management system that should be taken into consideration when analysing the environmental impacts. ${ }^{2-8}$ In the analyses on environment impact of the waste management system, the collection is either ignored or its role is exaggerated. ${ }^{9-11}$ Some authors claim that fuel consumption within a waste collection system, measured per mass unit of collected waste, has significant impact on the environment. ${ }^{12,13}$ Reduction of both the mass of waste transported and the transport distance

* Corresponding author: Dr. sc. Anamarija Grbeš

e-pošta: anamarija.grbes@rgn.hr is essential for reduction of the waste management impact on the environment. ${ }^{14}$

Waste removal (collection and landfilling) in the Republic of Croatia is the responsibility of the municipalities and local governments in 21 administrative units (counties). They entrust the respective economic activity to 208 private and public companies specialized in waste collection and treatment. Organised waste collection affects $99 \%$ of the population. The mixed waste from households and enterprises is at various frequencies collected at the door (kerbside collection) and transported by truck to a landfill, or, rarely, to a processing plant. The average annual mass of municipal solid waste per capita totals $382 \mathrm{~kg}$ (in the year 2014). The national municipal waste recycling rate amounts to $15 \%$ (in the year 2013), with most of the waste being landfilled. ${ }^{15}$

The main constituent of the fuel-related airborne emissions is carbon dioxide. In the literature, carbon dioxide emissions per ton of municipal solid waste that is related to its collection and transport, varies greatly. These differences arise from the differences in:

- applied waste collection technology: type of trucks, differences in fuel and fuel consumption;

- applied waste management methods in the studied area: kerbside collection of mixed or separated waste, transport to landfill or to treatment/recycling/incineration centre first; 
- method of data collection: direct measurement, average data;

- method of calculation of the $\mathrm{CO}_{2}$ emission - impact analysis applied: complete combustion of fuel into $\mathrm{CO}_{2}$ or incomplete combustion of fuel resulting in emission of $\mathrm{CO}_{2}$ and $\mathrm{CO}$, fuel combustion related emission, fuel life cycle related emission (including exploitation, refinery, regional storage).

The AEA study $(2001)^{16}$ gives the emission factors for collection and transport of waste for different treatment options. The factors are resulting from general fixed assumptions on vehicle types used, payloads and $\mathrm{km}$ travelled. The standard emission factor for transport of collected municipal solid waste (MSW) is $7 \mathrm{~kg}$ of fossil $\mathrm{CO}_{2}$ equivalent per ton of MSW. ${ }^{16}$ For separately collected fractions, the factor ranges from 8 to $15 \mathrm{~kg} \mathrm{CO}_{2}$ (eq) per ton of waste (Table 1). The same factors are used in JASPERS Knowledge Economy and Energy Division Staff for Calculation of GHG emissions of Waste Management Projects. ${ }^{17}$ Fig. 1 shows the waste management models in the AEA study, and the transport details of that model can be found in Appendix 1. Table A1.24. pp. 87-88 of the AEA study. ${ }^{16}$
Table 1 - Emission factors for collection and transport of waste for different treatment options ${ }^{16}$

Tablica 1 - Faktori emisija za sakupljanje i transport otpada za razne metode obrade otpada ${ }^{16}$

\begin{tabular}{|c|c|}
\hline Treatment option & $\begin{array}{c}\text { mass of } \mathrm{CO}_{2} \\
\text { equivalent } \\
\text { per mass of } \\
\mathrm{MSW} / \mathrm{kg} \mathrm{t}^{-1}\end{array}$ \\
\hline separately collected metal to sorting and recycling & 10 \\
\hline $\begin{array}{l}\text { separately collected plastic to sorting and } \\
\text { recycling }\end{array}$ & 15 \\
\hline $\begin{array}{l}\text { separately collected paper/cardboard to sorting } \\
\text { and recycling }\end{array}$ & 10 \\
\hline separately collected glass to sorting and recycling & 10 \\
\hline separately collected biowaste to composting & 8 \\
\hline separately collected biowaste to AD & 8 \\
\hline mixed waste to MBT & 9 \\
\hline mixed waste to incineration & 8 \\
\hline mixed waste to landfill & 7 \\
\hline
\end{tabular}

Table 2 - Breakdown of the $\mathrm{CO}_{2}$ emissions for the MRF scenario in Dresden (Winkler and Bilitewski, 2007, p. 1027) $)^{18}$

Tablica 2 - Pojedinačne emisije ugljikova dioksida iz sustava gospodarenja otpadom grada Dresdena izračunate u različitim programima (Winkler and Bilitewski, 2007, p. 1027) ${ }^{18}$

\begin{tabular}{l|r|r|c|c|c|c}
\hline & \multicolumn{6}{c}{$\mathrm{CO}_{2} / \mathrm{Mg}$} \\
\hline software & collection & \multicolumn{1}{c}{ MRF } & remanufacturing & transport & landfill & \multicolumn{1}{c}{ total } \\
\hline ARES & 233 & 11202 & 7597 & 188 & 44210 & 63,429 \\
EPIC/CSR & 368 & 3128 & -46451 & 75 & 44712 & 11832 \\
DST & 1639 & 11389 & -34676 & 538 & 32675 & 11566 \\
IWM2 & 416 & 3376 & -3271 & 0 & 24103 & 24624 \\
ORWARE & 740 & 12375 & -194223 & 567 & 54866 & -125674 \\
UMBERTO & 1278 & 446 & -276261 & 154 & 24965 & -249418 \\
\hline
\end{tabular}

Appendix 1. Mobilisation

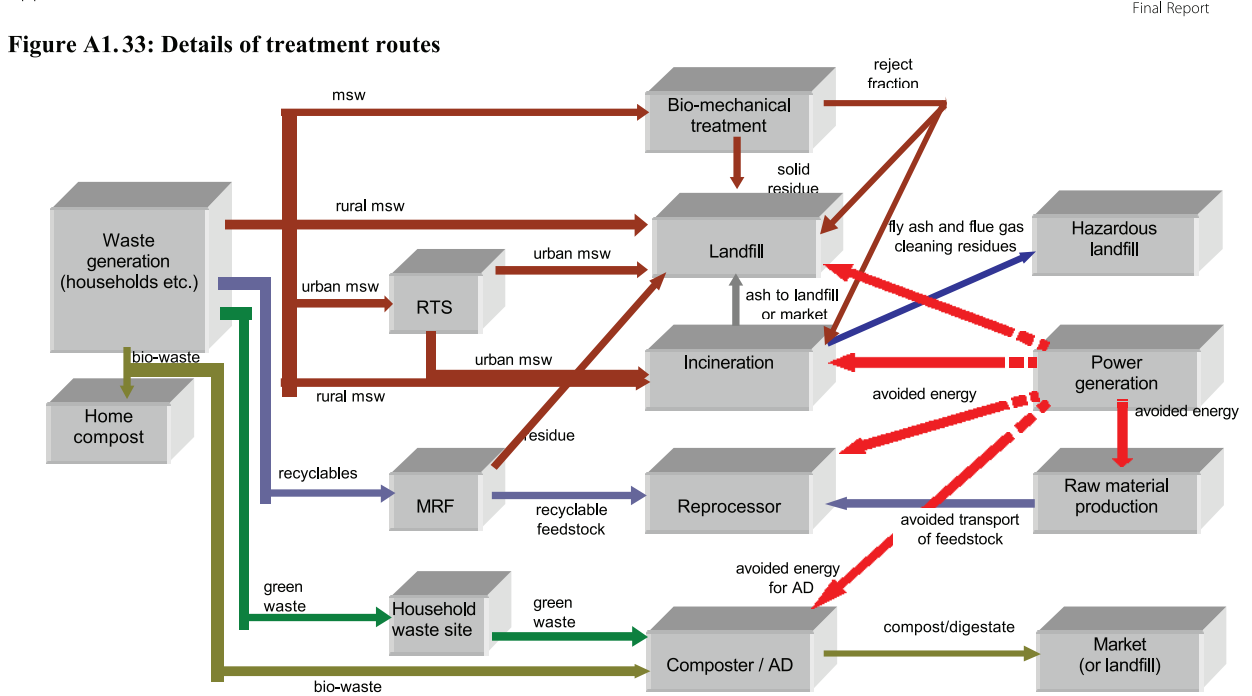

Fig. 1 - Details of treatment routes (AEA Technology, Final Report. Appendix 1. Mobilisation. Figure A1.33. p. 83) $)^{16}$ Slika 1 - Detalji metoda obrade otpada za koje su izračunati faktori emisija (AEA Technology, Final Report. Appendix 1. Mobilisation. Figure A1.33. str. 83) ${ }^{16}$ 
Winkler and Bilitewski (2007) $)^{18}$ performed a comparative evaluation of different LCA models for analysis of solid waste management in Dresden based on data for 1999. Dresden, a city with a population of approx. 1.2 million, produced that year 215420 t of solid waste from households and small businesses. The waste management of Dresden is shown in three scenarios: landfill, material recovery, and incineration. All scenarios assumed kerbside waste collection in bin and transport to the waste treatment facility in the waste collection truck. From their article, indicative is the table with $\mathrm{CO}_{2}$ emissions calculated for the materials recovery facility (MRF) scenario (Table 2). Their results show that total emission of $\mathrm{CO}_{2}$ from waste collection ranges from 233 to1639 $\mathrm{Mg} \mathrm{CO}_{2}\left(\mathrm{t} \mathrm{CO}_{2}\right){ }^{18}$

Hauser (2015) ${ }^{19}$ evaluated air emissions from trucks for solid waste management. The research was performed for a typical collection route in the Raleigh area, North Carolina. Total number of trucks in research was twenty-four. Fig. 2 shows the fuel consumption (diesel or compressed natural gas), and air emissions of carbon dioxide $\left(\mathrm{CO}_{2}\right)$, carbon monoxide $(\mathrm{CO})$, hydrocarbon $(\mathrm{HC})$, nitrous oxide $\left(\mathrm{NO}_{x}\right)$, and particulate matter (PM) per ton of waste collected in five types of WM trucks. The results of Hauser (2015) show diesel use of approx. 3.9-28.7 $\mathrm{kg} \mathrm{t}^{-1}$; emissions $\mathrm{CO}_{2}$ 12.6-90.0 kg t${ }^{-1}, \mathrm{CO} 29.0-434.4 \mathrm{~kg} \mathrm{t}^{-1}, \mathrm{HC} 8-38 \mathrm{gt}^{-1}$, $\mathrm{NO}_{x} 78-460 \mathrm{gt}^{-1}$, and PM $11 \mathrm{mgt}^{-1}-14 \mathrm{gt}^{-1}$. The calculated models represent the average fuel economy of trucks based on truck data that were recorded for every second spent in route. ${ }^{19}$

Salhofer et al. (2007) ${ }^{20}$ report global warming potential (GWP, kilograms of $\mathrm{CO}_{2}$ ), eutrophication (EP, grams of $\mathrm{PO}_{4}$ ), acidification (AP, grams of $\mathrm{SO}_{2}$ ), photochemical ozone - summer smog creation (POCP, grams of $\mathrm{C}_{2} \mathrm{H}_{4}$ ),

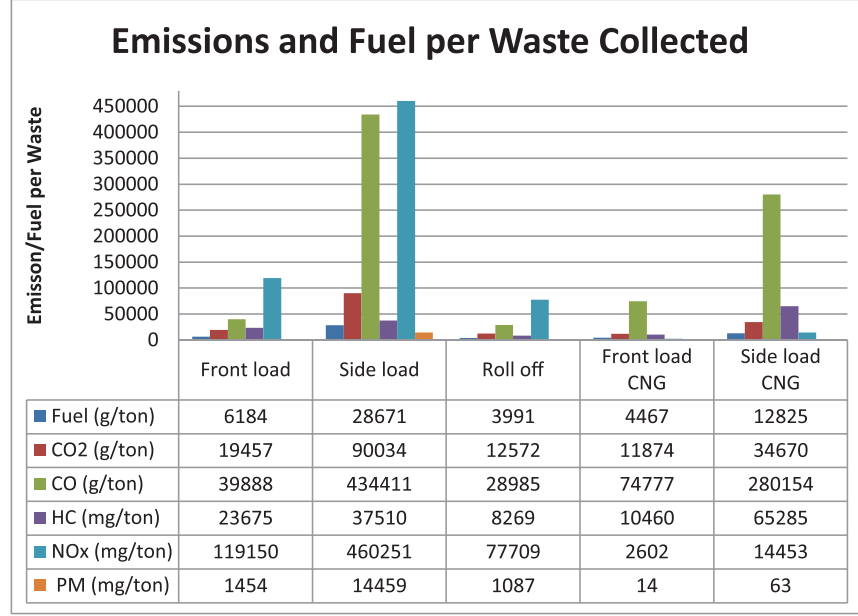

Fig. 2 - Emissions and fuel per waste collected per truck (Hauser, 2015, p. 52) ${ }^{19}$

Slika 2 - Emisije i gorivo po sakupljenom otpadu po vozilu (Hauser, 2015., str. 52) ${ }^{19}$

human toxicity (HTP, grams of DCB), and cumulated fossil energy demand $\left(\mathrm{CED}_{\mathrm{fos}}\right)$ for various fractions of separately collected waste at kerbside in three waste management scenarios: material recycling, thermal recycling, and disposal (Table 3). The scenarios represent the Austrian transport values for fractions of waste. The emission of $\mathrm{CO}_{2}$ (GWP) ranged from 12 to $703 \mathrm{~kg} \mathrm{t}^{-1}$ of waste: refrigerators 171-178, paper 13-22, polyethylene (PE) films 12-137, and expanded polystyrene (EPS) 12-703 kilograms of $\mathrm{CO}_{2}$ per ton of transported waste. For the refrigerators, the ozone depletion potential (ODP) in transport was also reported (16 g CFC11). ${ }^{20}$

Table 3 - Results of impact analysis for waste collection system (S. Salhofer et al., 2007) ${ }^{20}$

Tablica 3 - Rezultati analize utjecaja za sustav sakupljanja otpada (S. Salhofer et al., 2007) ${ }^{20}$

\begin{tabular}{|c|c|c|c|c|c|c|c|c|}
\hline Indicator & \multicolumn{4}{|c|}{ GWP (kilograms of $\mathrm{CO}_{2}$ ) } & \multicolumn{4}{|c|}{$\mathrm{EP}\left(\right.$ grams of $\mathrm{PO}_{4}$ ) } \\
\hline waste scenario & refrigerators & paper & PE films & EPS & refrigerators & paper & PE films & EPS \\
\hline material recycling & 178 & 22 & 137 & 703 & 6 & 23 & 142 & 783 \\
\hline thermal recycling & - & 20 & 106 & - & - & 22 & 107 & 803 \\
\hline \multirow[t]{2}{*}{ disposal } & 171 & 13 & 12 & 12 & 0 & 7 & 11 & 11 \\
\hline & \multicolumn{4}{|c|}{$\mathrm{AP}$ (grams of $\mathrm{SO}_{2}$ ) } & \multicolumn{4}{|c|}{$\mathrm{POCP}\left(\right.$ grams of $\left.\mathrm{C}_{2} \mathrm{H}_{4}\right)$} \\
\hline waste scenario & refrigerators & paper & PE films & EPS & refrigerators & paper & PE films & EPS \\
\hline material recycling & 8 & 24 & 153 & 763 & 1 & 2 & 15 & 65 \\
\hline thermal recycling & - & 22 & 122 & - & - & 2 & 13 & - \\
\hline disposal & 0 & 7 & 14 & 14 & 0 & 1 & 1 & 1 \\
\hline Indicator & \multicolumn{4}{|c|}{ HTP (grams of DCB) } & \multicolumn{4}{|c|}{$\mathrm{CED}_{\text {fos }}$} \\
\hline waste scenario & refrigerators & paper & PE films & EPS & refrigerators & paper & PE films & EPS \\
\hline material recycling & 421 & 1908 & 12906 & 53855 & 184 & 537 & 3437 & 17447 \\
\hline thermal recycling & - & 1779 & 11482 & - & - & 310 & 2685 & - \\
\hline disposal & 11 & 1321 & 1402 & 1402 & 4 & 176 & 298 & 298 \\
\hline
\end{tabular}




\section{Experimental}

\section{(mathematical formulas, tables \& equations)}

\subsection{Input data}

The input data for the analysis are based on the research of Ljubić (2015) and raw data tables from the Croatian environmental protection agency. Ljubić collected data from the waste collection companies (by phone and e-mail). Half of the 208 companies responded and delivered information on diesel consumption in 2013. Some companies delivered information on diesel consumption in volume unit litres, others in money (HRK) spent for the diesel with VAT (value added tax) included or excluded, some delivered information in both, litres and HRK. ${ }^{14}$
In this research, raw data retrieved from Ljubić ${ }^{4}$ are corrected. Firstly, the companies that were non-existent in the table received from the Croatian environmental protection agency are excluded because they were not operating in 2013. Secondly, the diesel volume is calculated from prices using the annual average price of diesel. Thus, the diesel figures presented here (Table 4) are somewhat different.

The Croatian environmental protection agency delivered raw data in an Excel document with registry of all the companies, counties they operate, and quantity and type of waste they collected. From that file, the quantity of waste in each county by type and total were summed. A copy was then made and all data for companies which had not delivered fuel data were deleted. That resulted with the

Table 4 - Diesel consumption in MSW collection system in Croatia 2013 and data representativeness

Tablica 4 - Prikupljeni podatci o potrošnji dizela u sustavu sakupljanja komunalnog otpada u Republici Hrvatskoj u 2013. i reprezentativnost podataka

\begin{tabular}{|c|c|c|c|c|c|c|c|c|}
\hline \multirow{3}{*}{ Administrative unit } & \multirow{3}{*}{ Population } & \multirow{2}{*}{\multicolumn{2}{|c|}{$\begin{array}{l}\text { Delivered data on } \\
\text { diesel consumed }\end{array}$}} & \multirow{3}{*}{ 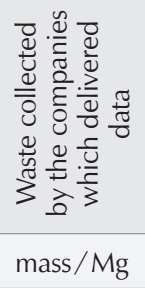 } & \multirow{3}{*}{\begin{tabular}{|l}
$\begin{array}{c}\text { Total waste } \\
\text { collected }\end{array}$ \\
mass / Mg \\
\end{tabular}} & \multirow{3}{*}{ 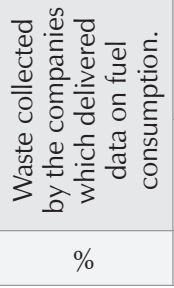 } & \multicolumn{2}{|c|}{$\begin{array}{l}\text { Structure of waste collected } \\
\text { by the companies which delivered data }\end{array}$} \\
\hline & & & & & & & $\begin{array}{l}\text { Mixed municipal } \\
\text { waste }\end{array}$ & $\begin{array}{c}\text { other municipal waste } \\
\text { (paper. glass. plastics. } \\
\text { bulky. packages....) }\end{array}$ \\
\hline & & volume $/ \mathrm{dm}^{3}$ & mass $/ \mathrm{kg}$ & & & & $\%$ & $\%$ \\
\hline Bjelovarsko-bilogorska & 104416 & 128582 & 106980 & 20778 & 25309 & 82.10 & 97.12 & 2.88 \\
\hline Brodsko-posavska & 158567 & 62916 & 52346 & 9241 & 45277 & 20.41 & 100.00 & 0.00 \\
\hline Dubrovačko-neretvanska & 123043 & 60473 & 50314 & 9069 & 63184 & 14.35 & 98.70 & 1.30 \\
\hline Grad Zagreb & 780000 & 303 & 252 & 54 & 332047 & 0.02 & 6.56 & 93.44 \\
\hline Istarska & 206286 & 417690 & 347518 & 85648 & 115732 & 74.01 & 88.10 & 11.90 \\
\hline Karlovačka & 125532 & 102398 & 85195 & 19899 & 43957 & 45.27 & 83.16 & 16.84 \\
\hline Koprivničko-križevačka & 112782 & 138340 & 115099 & 18801 & 25075 & 74.98 & 86.35 & 13.65 \\
\hline Krapinsko-zagorska & 126724 & 141528 & 117751 & 16400 & 65293 & 25.12 & 90.83 & 9.17 \\
\hline Ličko-senjska & 49700 & 93112 & 77469 & 8764 & 22837 & 38.37 & 97.51 & 2.49 \\
\hline Međimurska & 113197 & 69000 & 57408 & 14522 & 29338 & 49.50 & 70.91 & 29.09 \\
\hline Osječko-baranjska & 300959 & 550899 & 458348 & 67695 & 97881 & 69.16 & 96.64 & 3.36 \\
\hline Požeško-slavonska & 66995 & 83945 & 69842 & 13250 & 13796 & 96.04 & 94.54 & 5.46 \\
\hline Primorsko-goranska & 295988 & 730319 & 607625 & 84064 & 125357 & 67.06 & 72.96 & 27.04 \\
\hline Sisačko-moslavačka & 150696 & 193585 & 161063 & 27462 & 53186 & 51.63 & 95.24 & 4.76 \\
\hline Splitsko-dalmatinska & 454936 & 504732 & 419937 & 113508 & 214875 & 52.82 & 96.29 & 3.71 \\
\hline Šibensko-kninska & 109345 & 78800 & 65561 & 14883 & 49717 & 29.94 & 98.74 & 1.26 \\
\hline Varaždinska & 162761 & 64804 & 53917 & 10725 & 73158 & 14.66 & 92.43 & 7.57 \\
\hline Virovitičko-podravska & 84449 & 9952 & 8280 & 1958 & 24222 & 8.08 & 98.67 & 1.33 \\
\hline Vukovarsko-srijemska & 173760 & 114032 & 94875 & 26313 & 52888 & 49.75 & 97.29 & 2.71 \\
\hline Zadarska & 169165 & 710110 & 590811 & 85286 & 93163 & 91.54 & 92.37 & 7.63 \\
\hline Zagrebačka & 325226 & 40385 & 33600 & 12469 & 144797 & 8.61 & 76.60 & 23.40 \\
\hline MIN & 49700 & 303 & 252 & 54 & 13796 & 0.02 & 6.56 & 0.00 \\
\hline MAX & 780000 & 730319 & 607625 & 113508 & 332047 & 96.04 & 100.00 & 93.44 \\
\hline AVERAGE & 199739 & 204567 & 170200 & 31466 & 81480 & 45.88 & 87.19 & 12.81 \\
\hline CROATIA & 4194527 & 4295904 & 3574192 & 660790 & 1711089 & 38.62 & 90.13 & 9.87 \\
\hline
\end{tabular}


waste figures for companies which delivered data on fuel consumption.

Data representativeness for the county-level was calculated from the ratio of waste collected by the companies which delivered data on fuel consumption (column 7 of 9 in Table 4, and column 20 of 20 in Table 5).
Considering that the companies were unable to deliver the data on fuel consumption per waste stream collected, it was necessary to inspect for which type of waste the data is representative. That was calculated from the ratios of waste types in total waste collected by the companies which delivered data (summary in columns 8 and 9 in Table 4, detailed ratios in Table 6).

Table 5 - Percentage of waste in administrative unit collected by companies which delivered data on fuel consumption (by waste type)

Tablica 5 - Udio tvrtki - sakupljača otpada (koji su dostavili podatke o potrošnji goriva) u ukupno sakupljenom otpadu u administrativnoj jedinici (po vrsti otpada) u Republici Hrvatskoj u 2013.

\begin{tabular}{|c|c|c|c|c|c|c|c|c|c|c|c|c|c|c|c|c|c|c|c|}
\hline \multirow{2}{*}{$\begin{array}{l}\text { Administrative } \\
\text { unit (county) }\end{array}$} & \multicolumn{19}{|c|}{${ }^{*}$ Type of waste collected /\% } \\
\hline & A & B & C & D & $\mathrm{E}$ & $\mathrm{F}$ & G & $\mathrm{H}$ & 1 & J & K & $\mathrm{L}$ & $M$ & $\mathrm{~N}$ & $\mathrm{O}$ & $\mathrm{P}$ & Q & $\mathrm{R}$ & Sum \\
\hline $\begin{array}{l}\text { Bjelovarsko- } \\
\text { bilogorska }\end{array}$ & 0.00 & 0.00 & 0.00 & 0.00 & 0.10 & 0.00 & 0.01 & 79.73 & 0.00 & 1.05 & 0.00 & 0.15 & 0.84 & 0.00 & 0.00 & 0.00 & 0.20 & 0.00 & 82.10 \\
\hline $\begin{array}{l}\text { Brodsko- } \\
\text { posavska }\end{array}$ & 0.00 & 0.00 & 0.00 & 0.00 & 0.00 & 0.00 & 0.00 & 20.41 & 0.00 & 0.00 & 0.00 & 0.00 & 0.00 & 0.00 & 0.00 & 0.00 & 0.00 & 0.00 & 20.41 \\
\hline $\begin{array}{l}\text { Dubrovačko- } \\
\text { neretvanska }\end{array}$ & 0.00 & 0.00 & 0.00 & 0.00 & 0.00 & 0.19 & 0.00 & 14.17 & 0.00 & 0.00 & 0.00 & 0.00 & 0.00 & 0.00 & 0.00 & 0.00 & 0.00 & 0.00 & 14.35 \\
\hline Grad Zagreb & 0.00 & 0.00 & 0.00 & 0.00 & 0.00 & 0.00 & 0.00 & 0.00 & 0.00 & 0.00 & 0.00 & 0.00 & 0.00 & 0.00 & 0.00 & 0.00 & 0.00 & 0.02 & 0.02 \\
\hline Istarska & 0.02 & 1.19 & 0.05 & 0.00 & 3.75 & 0.94 & 0.00 & 65.20 & 0.00 & 1.32 & 0.35 & 0.42 & 0.40 & 0.00 & 0.09 & 0.22 & 0.04 & 0.00 & 74.01 \\
\hline Karlovačka & 0.00 & 0.02 & 0.00 & 0.00 & 0.05 & 5.30 & 0.00 & 37.64 & 0.00 & 1.86 & 0.25 & 0.00 & 0.00 & 0.00 & 0.05 & 0.00 & 0.00 & 0.09 & 45.27 \\
\hline $\begin{array}{l}\text { Koprivničko- } \\
\text { križevačka }\end{array}$ & 0.04 & 0.27 & 3.08 & 0.00 & 5.37 & 0.35 & 0.00 & 64.75 & 0.00 & 0.04 & 0.18 & 0.05 & 0.18 & 0.00 & 0.03 & 0.37 & 0.06 & 0.22 & 74.98 \\
\hline $\begin{array}{l}\text { Krapinsko- } \\
\text { zagorska }\end{array}$ & 0.00 & 0.03 & 0.00 & 0.00 & 0.61 & 1.62 & 0.00 & 22.81 & 0.00 & 0.00 & 0.00 & 0.00 & 0.00 & 0.00 & 0.00 & 0.03 & 0.00 & 0.00 & 25.12 \\
\hline Ličko-senjska & 0.00 & 0.60 & 0.22 & 0.00 & 0.00 & 0.00 & 0.00 & 37.42 & 0.00 & 0.00 & 0.00 & 0.00 & 0.00 & 0.00 & 0.03 & 0.11 & 0.00 & 0.00 & 38.37 \\
\hline Međimurska & 0.23 & 2.99 & 2.28 & 0.00 & 6.17 & 1.71 & 0.00 & 35.10 & 0.00 & 0.00 & 0.15 & 0.00 & 0.00 & 0.00 & 0.00 & 0.87 & 0.00 & 0.00 & 49.50 \\
\hline $\begin{array}{l}\text { Osječko- } \\
\text { baranjska }\end{array}$ & 0.00 & 0.12 & 0.02 & 0.00 & 0.02 & 0.29 & 0.04 & 66.84 & 0.00 & 0.00 & 0.01 & 0.00 & 1.62 & 0.00 & 0.11 & 0.09 & 0.00 & 0.00 & 69.16 \\
\hline $\begin{array}{l}\text { Požeško- } \\
\text { slavonska }\end{array}$ & 0.00 & 0.00 & 0.00 & 0.00 & 3.31 & 0.92 & 0.00 & 90.80 & 0.00 & 0.00 & 0.16 & 0.31 & 0.55 & 0.00 & 0.00 & 0.00 & 0.00 & 0.00 & 96.04 \\
\hline $\begin{array}{l}\text { Primorsko- } \\
\text { goranska }\end{array}$ & 0.02 & 2.68 & 0.59 & 0.00 & 4.71 & 7.87 & 0.00 & 48.93 & 0.00 & 0.74 & 0.18 & 0.43 & 0.31 & 0.00 & 0.04 & 0.56 & 0.00 & 0.00 & 67.06 \\
\hline $\begin{array}{l}\text { Sisačko- } \\
\text { moslavačka }\end{array}$ & 0.00 & 0.80 & 0.03 & 0.00 & 0.34 & 1.08 & 0.07 & 49.18 & 0.00 & 0.00 & 0.00 & 0.00 & 0.12 & 0.00 & 0.00 & 0.02 & 0.00 & 0.00 & 51.63 \\
\hline $\begin{array}{l}\text { Splitsko- } \\
\text { dalmatinska }\end{array}$ & 0.00 & 0.09 & 0.00 & 0.00 & 0.00 & 1.23 & 0.00 & 50.86 & 0.00 & 0.64 & 0.00 & 0.00 & 0.00 & 0.00 & 0.00 & 0.00 & 0.00 & 0.00 & 52.82 \\
\hline $\begin{array}{l}\text { Šibensko- } \\
\text { kninska }\end{array}$ & 0.00 & 0.27 & 0.00 & 0.00 & 0.00 & 0.06 & 0.00 & 29.56 & 0.00 & 0.00 & 0.00 & 0.00 & 0.04 & 0.00 & 0.00 & 0.00 & 0.00 & 0.00 & 29.94 \\
\hline Varaždinska & 0.00 & 0.10 & 0.04 & 0.00 & 0.74 & 0.03 & 0.00 & 13.55 & 0.00 & 0.00 & 0.01 & 0.00 & 0.15 & 0.00 & 0.00 & 0.04 & 0.00 & 0.00 & 14.66 \\
\hline $\begin{array}{l}\text { Virovitičko- } \\
\text { podravska }\end{array}$ & 0.00 & 0.00 & 0.00 & 0.00 & 0.00 & 0.11 & 0.00 & 7.98 & 0.00 & 0.00 & 0.00 & 0.00 & 0.00 & 0.00 & 0.00 & 0.00 & 0.00 & 0.00 & 8.08 \\
\hline $\begin{array}{l}\text { Vukovarsko- } \\
\text { srijemska }\end{array}$ & 0.00 & 0.00 & 0.00 & 0.00 & 0.05 & 0.61 & 0.00 & 48.40 & 0.00 & 0.34 & 0.00 & 0.00 & 0.00 & 0.00 & 0.00 & 0.00 & 0.00 & 0.35 & 49.75 \\
\hline Zadarska & 0.00 & 0.17 & 0.08 & 0.00 & 0.82 & 5.24 & 0.00 & 84.56 & 0.00 & 0.37 & 0.00 & 0.00 & 0.30 & 0.00 & 0.00 & 0.00 & 0.00 & 0.00 & 91.54 \\
\hline Zagrebačka & 0.00 & 0.00 & 0.00 & 0.00 & 0.01 & 0.13 & 0.00 & 6.60 & 0.00 & 0.00 & 0.00 & 0.00 & 0.02 & 0.00 & 0.00 & 0.01 & 0.01 & 1.84 & 8.61 \\
\hline MIN & 0.00 & 0.00 & 0.00 & 0.00 & 0.00 & 0.00 & 0.00 & 0.00 & 0.00 & 0.00 & 0.00 & 0.00 & 0.00 & 0.00 & 0.00 & 0.00 & 0.00 & 0.00 & 0.02 \\
\hline MAX & 0.23 & 2.99 & 3.08 & 0.00 & 6.17 & 7.87 & 0.07 & 90.80 & 0.00 & 1.86 & 0.35 & 0.43 & 1.62 & 0.00 & 0.11 & 0.87 & 0.20 & 1.84 & 96.04 \\
\hline AVERAGE & 0.02 & 0.54 & 0.41 & 0.00 & 1.40 & 1.55 & 0.01 & 41.97 & 0.00 & 0.36 & 0.07 & 0.08 & 0.27 & 0.00 & 0.02 & 0.14 & 0.02 & 0.19 & 46.06 \\
\hline CROATIA & 0.01 & 0.41 & 0.14 & 0.00 & 0.93 & 1.41 & 0.00 & 34.81 & 0.00 & 0.32 & 0.05 & 0.07 & 0.19 & 0.00 & 0.02 & 0.09 & 0.01 & 0.18 & 38.62 \\
\hline
\end{tabular}

* A - Metal packaging; B - Paper and cardboard packaging; C - Plastic packaging; D - Batteries and accumulators covered by 160601.1606 02 or 160603 and unassembled batteries and accumulators containing these batteries; E- Biodegradable waste; F - Bulky waste; G - Used tires; $\mathrm{H}$ - Mixed municipal waste; I - Discarded equipment containing chlorofluorocarbons. HCFCs. HFCs; J - Street cleaning residue; K - Other nonbiodegradable waste; $\mathrm{L}$ - Waste from the market; $\mathrm{M}$ - Paper and cardboard; $\mathrm{N}$ - Ashes from the litter grate. precipitate and dust from the boiler (other than the dust from the boiler mentioned under 1001 04); O - Plastics; P - Glass packaging; Q - Glass; R - Soil and rock 
Table 6 - Waste stream representativeness of delivered data on fuel consumption

Tablica 6 - Reprezentativnost dostavljenih podataka o potrošnji goriva za sakupljanje otpada u Republici Hrvatskoj u 2013. godini po vrstama otpada

\begin{tabular}{|c|c|c|c|c|c|c|c|c|c|c|c|c|c|c|c|c|c|c|c|}
\hline \multirow{2}{*}{$\begin{array}{l}\text { Administrative } \\
\text { unit (county) }\end{array}$} & \multicolumn{19}{|c|}{${ }^{*}$ Type of waste collected /\% } \\
\hline & A & B & C & $\mathrm{D}$ & $\mathrm{E}$ & $\mathrm{F}$ & G & $\mathrm{H}$ & I & $J$ & $\mathrm{~K}$ & $\mathrm{~L}$ & $M$ & $\mathrm{~N}$ & $\mathrm{O}$ & $\mathrm{P}$ & $\mathrm{Q}$ & $\mathrm{R}$ & Sum \\
\hline $\begin{array}{l}\text { Bjelovarsko- } \\
\text { bilogorska }\end{array}$ & 0.00 & 0.00 & 0.00 & 0.00 & 0.12 & 0.00 & 0.02 & 97.12 & 0.00 & 1.27 & 0.00 & 0.18 & 1.03 & 0.00 & 0.00 & 0.00 & 0.24 & 0.00 & 100.00 \\
\hline $\begin{array}{l}\text { Brodsko- } \\
\text { posavska }\end{array}$ & 0.00 & 0.00 & 0.00 & 0.00 & 0.00 & 0.00 & 0.00 & 100.00 & 0.00 & 0.00 & 0.00 & 0.00 & 0.00 & 0.00 & 0.00 & 0.00 & 0.00 & 0.00 & 100.00 \\
\hline $\begin{array}{l}\text { Dubrovačko- } \\
\text { neretvanska }\end{array}$ & 0.00 & 0.00 & 0.00 & 0.00 & 0.00 & 1.30 & 0.00 & 98.70 & 0.00 & 0.00 & 0.00 & 0.00 & 0.00 & 0.00 & 0.00 & 0.00 & 0.00 & 0.00 & 100.00 \\
\hline Grad Zagreb & 0.00 & 0.00 & 0.00 & 0.00 & 0.00 & 0.00 & 0.00 & 6.56 & 0.00 & 0.00 & 0.00 & 0.00 & 0.00 & 0.00 & 0.00 & 0.00 & 0.00 & 93.44 & 100.00 \\
\hline Istarska & 0.02 & 1.61 & 0.07 & 0.00 & 5.07 & 1.27 & 0.00 & 88.10 & 0.00 & 1.78 & 0.48 & 0.57 & 0.54 & 0.00 & 0.12 & 0.30 & 0.05 & 0.00 & 100.00 \\
\hline Karlovačka & 0.00 & 0.04 & 0.00 & 0.00 & 0.11 & 11.72 & 0.00 & 83.16 & 0.00 & 4.10 & 0.55 & 0.00 & 0.01 & 0.00 & 0.11 & 0.00 & 0.00 & 0.20 & 100.00 \\
\hline $\begin{array}{l}\text { Koprivničko- } \\
\text { križevačka }\end{array}$ & 0.05 & 0.36 & 4.11 & 0.00 & 7.17 & 0.46 & 0.00 & 86.35 & 0.00 & 0.05 & 0.23 & 0.07 & 0.24 & 0.00 & 0.04 & 0.49 & 0.08 & 0.29 & 100.00 \\
\hline $\begin{array}{l}\text { Krapinsko- } \\
\text { zagorska }\end{array}$ & 0.00 & 0.13 & 0.01 & 0.00 & 2.45 & 6.45 & 0.00 & 90.83 & 0.00 & 0.00 & 0.00 & 0.00 & 0.00 & 0.00 & 0.00 & 0.14 & 0.00 & 0.00 & 100.00 \\
\hline Ličko-senjska & 0.00 & 1.56 & 0.58 & 0.00 & 0.00 & 0.00 & 0.00 & 97.51 & 0.00 & 0.00 & 0.00 & 0.00 & 0.00 & 0.00 & 0.07 & 0.28 & 0.00 & 0.00 & 100.00 \\
\hline Međimurska & 0.46 & 6.04 & 4.60 & 0.00 & 12.46 & 3.46 & 0.00 & 70.91 & 0.00 & 0.00 & 0.30 & 0.00 & 0.00 & 0.00 & 0.00 & 1.77 & 0.01 & 0.00 & 100.00 \\
\hline $\begin{array}{l}\text { Osječko- } \\
\text { baranjska }\end{array}$ & 0.00 & 0.18 & 0.02 & 0.00 & 0.03 & 0.42 & 0.06 & 96.64 & 0.00 & 0.00 & 0.01 & 0.00 & 2.34 & 0.00 & 0.17 & 0.14 & 0.00 & 0.00 & 100.00 \\
\hline $\begin{array}{l}\text { Požeško- } \\
\text { slavonska }\end{array}$ & 0.00 & 0.00 & 0.00 & 0.00 & 3.44 & 0.96 & 0.00 & 94.54 & 0.00 & 0.00 & 0.17 & 0.33 & 0.57 & 0.00 & 0.00 & 0.00 & 0.00 & 0.00 & 100.00 \\
\hline $\begin{array}{l}\text { Primorsko- } \\
\text { goranska }\end{array}$ & 0.03 & 4.00 & 0.88 & 0.00 & 7.02 & 11.74 & 0.00 & 72.96 & 0.00 & 1.11 & 0.27 & 0.63 & 0.45 & 0.00 & 0.06 & 0.84 & 0.01 & 0.00 & 100.00 \\
\hline $\begin{array}{l}\text { Sisačko- } \\
\text { moslavačka }\end{array}$ & 0.00 & 1.55 & 0.07 & 0.00 & 0.66 & 2.09 & 0.13 & 95.24 & 0.00 & 0.00 & 0.00 & 0.00 & 0.23 & 0.00 & 0.00 & 0.04 & 0.00 & 0.00 & 100.00 \\
\hline $\begin{array}{l}\text { Splitsko- } \\
\text { dalmatinska }\end{array}$ & 0.00 & 0.18 & 0.00 & 0.00 & 0.00 & 2.33 & 0.00 & 96.29 & 0.00 & 1.20 & 0.00 & 0.00 & 0.00 & 0.00 & 0.00 & 0.00 & 0.00 & 0.00 & 100.00 \\
\hline $\begin{array}{l}\text { Šibensko- } \\
\text { kninska }\end{array}$ & 0.00 & 0.92 & 0.00 & 0.00 & 0.00 & 0.19 & 0.00 & 98.74 & 0.00 & 0.00 & 0.00 & 0.00 & 0.15 & 0.00 & 0.00 & 0.00 & 0.00 & 0.00 & 100.00 \\
\hline Varaždinska & 0.00 & 0.70 & 0.29 & 0.00 & 5.05 & 0.19 & 0.00 & 92.43 & 0.00 & 0.00 & 0.04 & 0.00 & 1.00 & 0.00 & 0.00 & 0.30 & 0.00 & 0.00 & 100.00 \\
\hline $\begin{array}{l}\text { Virovitičko- } \\
\text { podravska }\end{array}$ & 0.00 & 0.00 & 0.00 & 0.00 & 0.00 & 1.33 & 0.00 & 98.67 & 0.00 & 0.00 & 0.00 & 0.00 & 0.00 & 0.00 & 0.00 & 0.00 & 0.00 & 0.00 & 100.00 \\
\hline $\begin{array}{l}\text { Vukovarsko- } \\
\text { srijemska }\end{array}$ & 0.00 & 0.00 & 0.00 & 0.00 & 0.10 & 1.22 & 0.00 & 97.29 & 0.00 & 0.69 & 0.00 & 0.00 & 0.00 & 0.00 & 0.00 & 0.00 & 0.00 & 0.70 & 100.00 \\
\hline Zadarska & 0.00 & 0.19 & 0.08 & 0.00 & 0.90 & 5.73 & 0.00 & 92.37 & 0.00 & 0.40 & 0.00 & 0.00 & 0.33 & 0.00 & 0.00 & 0.00 & 0.00 & 0.00 & 100.00 \\
\hline Zagrebačka & 0.00 & 0.00 & 0.00 & 0.00 & 0.12 & 1.47 & 0.00 & 76.60 & 0.00 & 0.00 & 0.00 & 0.00 & 0.21 & 0.00 & 0.00 & 0.11 & 0.08 & 21.40 & 100.00 \\
\hline MIN & 0.00 & 0.00 & 0.00 & 0.00 & 0.00 & 0.00 & 0.00 & 6.56 & 0.00 & 0.00 & 0.00 & 0.00 & 0.00 & 0.00 & 0.00 & 0.00 & 0.00 & 0.00 & 100.00 \\
\hline MAX & 0.46 & 6.04 & 4.60 & 0.00 & 12.46 & 11.74 & 0.13 & 100.00 & 0.00 & 4.10 & 0.55 & 0.63 & 2.34 & 0.00 & 0.17 & 1.77 & 0.24 & 93.44 & 100.00 \\
\hline AVERAGE & 0.04 & 1.02 & 0.67 & 0.00 & 2.48 & 2.79 & 0.01 & 84.24 & 0.00 & 0.64 & 0.11 & 0.11 & 0.41 & 0.00 & 0.03 & 0.27 & 0.03 & 9.11 & 100.00 \\
\hline CROATIA & 0.05 & 1.07 & 0.69 & 0.00 & 2.59 & 2.91 & 0.01 & 83.68 & 0.00 & 0.61 & 0.12 & 0.10 & 0.38 & 0.00 & 0.03 & 0.28 & 0.02 & 9.50 & 100.00 \\
\hline
\end{tabular}

* A - Metal packaging; B - Paper and cardboard packaging; C - Plastic packaging; D - Batteries and accumulators covered by 160601.1606 02 or 160603 and unassembled batteries and accumulators containing these batteries; E- Biodegradable waste; F - Bulky waste; G - Used tires; H - Mixed municipal waste; I - Discarded equipment containing chlorofluorocarbons. HCFCs. HFCs; J - Street cleaning residue; K - Other nonbiodegradable waste; $\mathrm{L}$ - Waste from the market; $\mathrm{M}$ - Paper and cardboard; $\mathrm{N}$ - Ashes from the litter grate. precipitate and dust from the boiler (other than the dust from the boiler mentioned under 1001 04); O - Plastics; P - Glass packaging; Q - Glass; R - Soil and rock 


\subsection{Calculation method for the diesel consumption estimate}

The data sample represents:

- $50 \%$ of the statistical population (i.e. more than 100 companies out of 208) approximately

- $39 \%$ of the waste collected in the Republic of Croatia in $2013,84 \%$ of which was mixed municipal waste

At the administrative unit level, data samples represent:

- $0-100 \%$ of the statistical population in a county, on average $50 \%$,

- $0.02-96.04 \%$ (on average $46.06 \%$ ) of the waste collected in a county in 2013,

- $6.56-100 \%$ (on average $84.24 \%$ ) of the collected waste was mixed municipal waste.

With assumptions that diesel consumption per ton of waste is county-specific and that the gathered data is sufficiently representative, the diesel consumption from the partial data for each county is up-scaled to the whole county. The structure of waste streams collected in each county in total, and by the companies that participated in the research are already shown in the previous section in Tables 4-6.

County-specific diesel consumption per ton of waste is calculated by dividing the data on diesel that the companies had delivered with the quantity of collected waste that those companies had reported. The specific diesel consumption is then multiplied with the total quantity of waste collected by all companies in the county, resulting in total waste collection related diesel consumption in a county.

The described procedure is applied to all counties except for the county of the City of Zagreb for which no relevant data is collected. For that county, the estimate is performed by multiplying the country-specific fuel consumption per ton of waste $\left(6,50 \mathrm{I} \mathrm{mg}^{-1} ; 5,41 \mathrm{~kg} \mathrm{mg}^{-1}\right)$ with the quantity of reported waste for the City of Zagreb.

\subsection{Emission factors for heavy duty diesel vehicles}

For the calculation of fuel combustion related emission, the Tier 1 emission factors for heavy duty diesel vehicles (Table 7) from the EMEP/EEA air pollutant emission inventory guidebook are used (EEA, 2016). ${ }^{21}$

\section{Results and discussion}

\subsection{Results}

Table 8 shows the diesel consumption estimate based on data delivered from $50 \%$ of the companies (up-scaled
Table 7 - Tier 1 emission factors for heavy duty diesel vehicles (from Tables 3.5-3.15 in EMEP/EEA air pollutant emission inventory guidebook 2016 - Update Dec. 2016) ${ }^{21}$

Tablica 7 - Faktori Tier 1 emisija za teška dizelska vozila (iz tablica 3.5 - 3.15 u EMEP/EEA air pollutant emission inventory guidebook 2016 - Update Dec. 2016) ${ }^{21}$

\begin{tabular}{|c|c|c|c|c|}
\hline Emission & Unit & Mean & Min & Max \\
\hline carbon dioxide, $\mathrm{CO}_{2}$ & $\mathrm{~kg} / \mathrm{kg}$ fuel & 3.18 & 3.18 & 3.18 \\
\hline nitrogen oxides, $\mathrm{NO}_{x}$ & $\mathrm{~g} / \mathrm{kg}$ fuel & 33.37 & 28.34 & 38.29 \\
\hline carbon monoxide, $\mathrm{CO}$ & $\mathrm{g} / \mathrm{kg}$ fuel & 7.58 & 5.73 & 10.57 \\
\hline $\begin{array}{l}\text { carbon dioxide from } \\
\text { lubricant, } \mathrm{CO}_{2}\end{array}$ & $\mathrm{~g} / \mathrm{kg}$ fuel & 2.54 & 1.99 & 3.32 \\
\hline $\begin{array}{l}\text { non-methane volatile } \\
\text { organic compounds, } \\
\text { NMVOC }\end{array}$ & $\mathrm{g} / \mathrm{kg}$ fuel & 1.92 & 1.33 & 3.77 \\
\hline particulate matter, PM & $\mathrm{g} / \mathrm{kg}$ fuel & 0.94 & 0.61 & 1.57 \\
\hline $\begin{array}{l}\text { black carbon fraction } \\
\text { of particulate matter, } \\
\text { f-BC, } 0.53\end{array}$ & $\mathrm{~g} / \mathrm{kg}$ fuel & 0.4982 & 0.3233 & 0.8321 \\
\hline nitrous oxide, $\mathrm{N}_{2} \mathrm{O}$ & $\mathrm{g} / \mathrm{kg}$ fuel & 0.051 & 0.03 & 0.089 \\
\hline $\begin{array}{l}\mathrm{SO}_{2} \text {, sulphur content } \\
\text { fuel } 20098 \mathrm{ppm}\end{array}$ & $\mathrm{mg} / \mathrm{kg}$ fuel & 16 & 16 & 16 \\
\hline ammonia, $\mathrm{NH}_{3}$ & $\mathrm{~g} / \mathrm{kg}$ fuel & 0.013 & 0.01 & 0.018 \\
\hline lead, $\mathrm{Pb}$ & $\mu \mathrm{g} / \mathrm{kg}$ fuel & 52 & 16 & 19.4 \\
\hline $\begin{array}{l}\text { indeno[1,2,3-cd }] \\
\text { pyrene, ID[1,2,3-cd]P }\end{array}$ & $\mu g / \mathrm{kg}$ fuel & 7.9 & 8.6 & 7.3 \\
\hline $\begin{array}{l}\text { benzo[k]fluoranthene, } \\
\mathrm{B}[\mathrm{k}] \mathrm{F}\end{array}$ & $\mu \mathrm{g} / \mathrm{kg}$ fuel & 34.4 & 37.2 & 31.8 \\
\hline $\begin{array}{l}\text { benzo[b]fluoranthene, } \\
\text { B[b]F }\end{array}$ & $\mu \mathrm{g} / \mathrm{kg}$ fuel & 30.8 & 33.3 & 28.4 \\
\hline benzo[a]pyrene, B[a]P & $\mu \mathrm{g} / \mathrm{kg}$ fuel & 5.1 & 5.5 & 4.7 \\
\hline $\begin{array}{l}\text { typical fuel } \\
\text { consumption }\end{array}$ & $\mathrm{g}$ fuel $/ \mathrm{km}$ & 240 & 240 & 240 \\
\hline
\end{tabular}

data), relative consumption per ton of waste and per capita in each county, and for the whole country. Tables 9 and 10 show the diesel related emissions to air from the waste collection system, for each county, and for the whole country.

Annual consumption of diesel in Croatian MSW collection systems in 2013 was 10.6 million litres or 8.8 thousand tons. At county level, this ranges from 87 thousand litres to 2.2 million litres (73-1796 t). Median value for consumption, at county level, was 375 thousand litres (312 t), average 503 thousand litres (419 t). Relative consumption of diesel from waste collection was on average $6.50 \mathrm{I}\left(5.41 \mathrm{~kg} \mathrm{t}^{-1}\right)$ of MSW (ranging from 3.24 to $10.62 \mathrm{l}$ ), 
Table 8 - Diesel consumption estimate for Croatian municipal solid waste collection system in 2013

Tablica 8 - Procjena potrošnje dizelskog goriva u sustavu sakupljanja komunalnog otpada u Hrvatskoj u 2013. godini

\begin{tabular}{|c|c|c|c|c|c|c|}
\hline \multirow{2}{*}{$\begin{array}{l}\text { Administrative unit } \\
\text { (county) }\end{array}$} & \multicolumn{2}{|c|}{$\begin{array}{l}\text { Diesel consumption } \\
\text { per mass of waste }\end{array}$} & \multicolumn{2}{|c|}{$\begin{array}{c}\text { Diesel consumption } \\
\text { per capita }\end{array}$} & \multicolumn{2}{|c|}{ Total diesel consumed } \\
\hline & $\mathrm{dm}^{3} / \mathrm{Mg}$ & $\mathrm{kg} / \mathrm{Mg}$ & $d m^{3}$ & $\mathrm{~kg}$ & $\mathrm{dm}^{3}$ & $\mathrm{~kg}$ \\
\hline Bjelovarsko-bilogorska & 6.19 & 5.15 & 1.50 & 1.25 & 156625 & 130312 \\
\hline Brodsko-posavska & 6.81 & 5.66 & 1.94 & 1.62 & 308272 & 256482 \\
\hline Dubrovačko-neretvanska & 6.67 & 5.55 & 3.42 & 2.85 & 421332 & 350548 \\
\hline Grad Zagreb & 6.50 & 5.41 & 2.77 & 2.30 & 2158690 & 1796030 \\
\hline Istarska & 4.88 & 4.06 & 2.74 & 2.28 & 564407 & 469587 \\
\hline Karlovačka & 5.15 & 4.28 & 1.80 & 1.50 & 226198 & 188197 \\
\hline Koprivničko-križevačka & 7.36 & 6.12 & 1.64 & 1.36 & 184503 & 153507 \\
\hline Krapinsko-zagorska & 8.63 & 7.18 & 4.45 & 3.70 & 563465 & 468803 \\
\hline Ličko-senjska & 10.62 & 8.84 & 4.88 & 4.06 & 242640 & 201876 \\
\hline Međimurska & 4.75 & 3.95 & 1.23 & 1.02 & 139390 & 115973 \\
\hline Osječko-baranjska & 8.14 & 6.77 & 2.65 & 2.20 & 796549 & 662728 \\
\hline Požeško-slavonska & 6.34 & 5.27 & 1.30 & 1.09 & 87403 & 72719 \\
\hline Primorsko-goranska & 8.69 & 7.23 & 3.68 & 3.06 & 1089056 & 906094 \\
\hline Sisačko-moslavačka & 7.05 & 5.86 & 2.49 & 2.07 & 374915 & 311929 \\
\hline Splitsko-dalmatinska & 4.45 & 3.70 & 2.10 & 1.75 & 955482 & 794961 \\
\hline Šibensko-kninska & 5.29 & 4.41 & 2.41 & 2.00 & 263229 & 219007 \\
\hline Varaždinska & 6.04 & 5.03 & 2.72 & 2.26 & 442024 & 367764 \\
\hline Virovitičko-podravska & 5.08 & 4.23 & 1.46 & 1.21 & 123109 & 102427 \\
\hline Vukovarsko-srijemska & 4.33 & 3.61 & 1.32 & 1.10 & 229201 & 190695 \\
\hline Zadarska & 8.33 & 6.93 & 4.59 & 3.82 & 775696 & 645379 \\
\hline Zagrebačka & 3.24 & 2.69 & 1.44 & 1.20 & 468962 & 390176 \\
\hline MIN & 3.24 & 2.69 & 1.23 & 1.02 & 87403 & 72719 \\
\hline MAX & 10.62 & 8.84 & 4.88 & 4.06 & 2158690 & 1796030 \\
\hline AVERAGE & 6.41 & 5.33 & 2.50 & 2.08 & 503388 & 418819 \\
\hline MEDIAN & 6.34 & 5.27 & 2.41 & 2.00 & 374915 & 311929 \\
\hline CROATIA & 6.50 & 5.41 & 2.52 & 2.10 & 10571147 & 8795195 \\
\hline
\end{tabular}

or 2.52 I per capita (1.23-4.88 I). Total consumption of diesel for MSW collection in Croatia is estimated at 10.6 million litres (Table 8). The data is graphically presented in Figs. 3-8.

Emissions at county level (Tables 9 and 10) are following the same trends as the diesel consumption data shown in Figs. 3, 4 and 5. Twelve counties have below-average relative diesel consumption per capita, and eleven counties have below-average relative diesel consumption per ton of MSW. Below the median value for diesel consumption per capita and per ton of MSW are counties nine and ten respectively (Figs. 3 and 4). The relative diesel consumption is the lowest in the continental counties, and highest in counties with mountainous terrain, such as Ličko-senjska, Primorsko-goranska, Krapinsko-zagorska and Zadarska.
Twelve counties have total diesel consumption below the median value, fourteen below the average value; most of them are the continental counties (Fig. 5). Total diesel consumption is the highest in the county of the City of Zagreb (Grad Zagreb), and the lowest in the county of Požega (Požeško-slavonska županija).

Carbon dioxide. Emission of carbon dioxide into the air from diesel combustion in Croatian MSW collection systems in 2013 ranged from 231 to 5711 t per county. Total annual emission of $\mathrm{CO}_{2}$ from MSW collection systems was $28000 \mathrm{t}$. Median value for $\mathrm{CO}_{2}$ emission at county level was $992 \mathrm{t}$, average $1332 \mathrm{t}$ (Table 9). Relative emission ranged from 3.26 to $13 \mathrm{~kg} \mathrm{CO}_{2}$ per capita (average $6 \mathrm{~kg}$ per capita), 
or $8.57-28.11 \mathrm{kgt}^{-1}$ of municipal waste (average $16.95 \mathrm{~kg}$ of $\mathrm{CO}_{2}$ per ton of municipal waste) (Appendices 1 and 2).

Nitrogen oxides. Emission of nitrogen oxides into the air from diesel combustion in Croatian MSW collection systems in 2013, at county level, ranged from 2.4 to $52 \mathrm{t}$. Total annual emission of $\mathrm{NO}_{x}$ from MSW collection systems was $284 \mathrm{t}$. Median value for $\mathrm{NO}_{x}$ emission at county level was $8.5 \mathrm{t}$, average $13.5 \mathrm{t}$ (Table 9). Relative emission ranged from 26 to $134 \mathrm{~g} \mathrm{NO}_{x}$ per capita (average $67 \mathrm{~g} \mathrm{NO}_{x}$ per capita), or $127-431 \mathrm{~g} \mathrm{t}^{-1}$ of municipal waste (average $231 \mathrm{~g}$ of $\mathrm{NO}_{x}$ per ton of municipal waste) (Appendices 1 and 2).

Carbon monoxide. Emission of carbon monoxide into the air from diesel combustion in Croatian MSW collection systems in 2013, at county level, ranged from 0.55 to $12 \mathrm{t}$. Total annual emission of CO from MSW collection systems was $12 \mathrm{t}$. Median value for $\mathrm{CO}$ emission at county level was $1.9 \mathrm{t}$, average $3 \mathrm{t}$ (Table 9). Relative emission ranged

Table 9 - Diesel related emissions to air from the Croatian municipal solid waste collection in 2013: $\mathrm{CO}_{2}, \mathrm{NO}_{x}, \mathrm{CO}_{1} \mathrm{CO}_{2}$ from lubricant, NMVOC, PM, f-BC and $\mathrm{N}_{2} \mathrm{O}$

Tablica 9 - Emisije u zrak vezane uz potrošnju dizelskog goriva u sustavu sakupljanja komunalnog otpada u Hrvatskoj u 2013. godini: $\mathrm{CO}_{2}, \mathrm{NO}_{x}, \mathrm{CO}, \mathrm{CO}_{2}$ od izgaranja maziva, $\mathrm{NMVOC}, \mathrm{PM}, \mathrm{f}-\mathrm{BC}$ i $\mathrm{N}_{2} \mathrm{O}$

\begin{tabular}{|c|c|c|c|c|c|c|c|c|}
\hline Administrative unit & $\mathrm{CO}_{2}$ & $\mathrm{NO}_{x}$ & $\mathrm{CO}$ & $\mathrm{CO}_{2}$-lub. & NMVOC & PM & $\mathrm{f}-\mathrm{BC}$ & $\mathrm{N}_{2} \mathrm{O}$ \\
\hline County of & $\mathrm{Mg}$ & $\mathrm{Mg}$ & $\mathrm{Mg}$ & $\mathrm{kg}$ & $\mathrm{kg}$ & $\mathrm{kg}$ & $\mathrm{kg}$ & $\mathrm{kg}$ \\
\hline Bjelovarsko-bilogorska & 414.39 & 4.35 & 0.99 & 330.99 & 250.20 & 122.49 & 64.92 & 6.65 \\
\hline Brodsko-posavska & 815.61 & 8.56 & 1.94 & 651.46 & 492.45 & 241.09 & 127.78 & 13.08 \\
\hline Dubrovačko-neretvanska & 1114.74 & 11.70 & 2.66 & 890.39 & 673.05 & 329.52 & 174.64 & 17.88 \\
\hline Grad Zagreb & 5711.38 & 59.93 & 13.61 & 4561.92 & 3448.38 & 1688.27 & 894.78 & 91.60 \\
\hline Istarska & 1493.29 & 15.67 & 3.56 & 1192.75 & 901.61 & 441.41 & 233.95 & 23.95 \\
\hline Karlovačka & 598.47 & 6.28 & 1.43 & 478.02 & 361.34 & 176.91 & 93.76 & 9.60 \\
\hline Koprivničko-križevačka & 488.15 & 5.12 & 1.16 & 389.91 & 294.73 & 144.30 & 76.48 & 7.83 \\
\hline Krapinsko-zagorska & 1490.79 & 15.64 & 3.55 & 1190.76 & 900.10 & 440.67 & 233.56 & 23.91 \\
\hline Ličko-senjska & 641.97 & 6.74 & 1.53 & 512.77 & 387.60 & 189.76 & 100.57 & 10.30 \\
\hline Međimurska & 368.79 & 3.87 & 0.88 & 294.57 & 222.67 & 109.01 & 57.78 & 5.91 \\
\hline Osječko-baranjska & 2107.48 & 22.12 & 5.02 & 1683.33 & 1272.44 & 622.96 & 330.17 & 33.80 \\
\hline Požeško-slavonska & 231.25 & 2.43 & 0.55 & 184.71 & 139.62 & 68.36 & 36.23 & 3.71 \\
\hline Primorsko-goranska & 2881.38 & 30.24 & 6.87 & 2301.48 & 1739.70 & 851.73 & 451.42 & 46.21 \\
\hline Sisačko-moslavačka & 991.93 & 10.41 & 2.36 & 792.30 & 598.90 & 293.21 & 155.40 & 15.91 \\
\hline Splitsko-dalmatinska & 2527.98 & 26.53 & 6.03 & 2019.20 & 1526.33 & 747.26 & 396.05 & 40.54 \\
\hline Šibensko-kninska & 696.44 & 7.31 & 1.66 & 556.28 & 420.49 & 205.87 & 109.11 & 11.17 \\
\hline Varaždinska & 1169.49 & 12.27 & 2.79 & 934.12 & 706.11 & 345.70 & 183.22 & 18.76 \\
\hline Virovitičko-podravska & 325.72 & 3.42 & 0.78 & 260.16 & 196.66 & 96.28 & 51.03 & 5.22 \\
\hline Vukovarsko-srijemska & 606.41 & 6.36 & 1.45 & 484.37 & 366.14 & 179.25 & 95.00 & 9.73 \\
\hline Zadarska & 2052.31 & 21.54 & 4.89 & 1639.26 & 1239.13 & 606.66 & 321.53 & 32.91 \\
\hline Zagrebačka & 1240.76 & 13.02 & 2.96 & 991.05 & 749.14 & 366.77 & 194.39 & 19.90 \\
\hline MIN & 231.25 & 2.43 & 0.55 & 184.71 & 139.62 & 68.36 & 36.23 & 3.71 \\
\hline MAX & 5711.38 & 59.93 & 13.61 & 4561.92 & 3448.38 & 1688.27 & 894.78 & 91.60 \\
\hline AVERAGE & 1331.84 & 13.98 & 3.17 & 1063.80 & 804.13 & 393.69 & 208.66 & 21.36 \\
\hline MEDIAN & 991.93 & 10.41 & 2.36 & 792.30 & 598.90 & 293.21 & 155.40 & 15.91 \\
\hline CROATIA & 27968.72 & 293.50 & 66.67 & 22339.79 & 16886.77 & 8267.48 & 4381.77 & 448.55 \\
\hline
\end{tabular}


Diesel consumption per mass of collected municipal waste in Croatian counties in $2013(\mathrm{~kg} / \mathrm{Mg})$

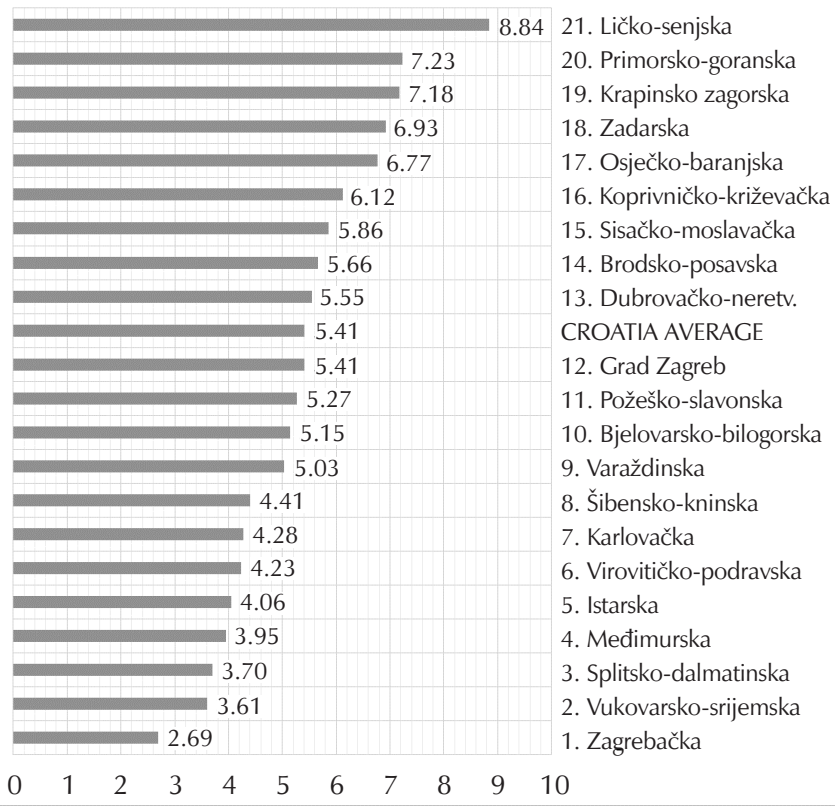

Fig. 3 -Relative diesel consumption per mass of collected mixed waste and per capita for mixed waste collection in Croatian counties in 2013

Slika 3 - Relativna potrošnja dizela u sustavu sakupljanja komunalnog otpada u Hrvatskoj u 2013. godini po megagramu (toni) otpada i po stanovniku županije

from 6 to 30 g CO per capita (average 15 g CO per capita or $29-98 \mathrm{gt}^{-1}$ of municipal waste (average 53 grams of $\mathrm{CO}$ per ton of municipal waste) (Appendices 1 and 2).

Carbon dioxide from lubricant. Emission of carbon dioxide into the air from engine lubricant in Croatian MSW collection systems in 2013, at county level, ranged from 0.2 to 4 t. Total annual emission of $\mathrm{CO}_{2}$ from lubricant in MSW collection systems was $22 \mathrm{t}$. Median value for engine lubricant $\mathrm{CO}_{2}$ emission at county level was $0.65 \mathrm{t}$ per county, average $1 \mathrm{t}$ (Table 9). Relative emission ranged from 2 to $10 \mathrm{~kg} \mathrm{CO}_{2}$ per capita (average $5 \mathrm{~g}$ per capita), or $10-33 \mathrm{~g} \mathrm{t}^{-1}$ of municipal waste (average $18 \mathrm{~g}$ of $\mathrm{CO}_{2}$ per ton of municipal waste) (Appendices 1 and 2).

Non-methane volatile organic compounds. Emission of non-methane volatile organic compounds into the air from diesel combustion in Croatian MSW collection systems in 2013, at county level, ranged from 0.14 to 3 t. Total annual emission of NMVOC from MSW collection systems was 16.4 t. Median value for NMVOC emission at county level was $0.5 \mathrm{t}$, average $0.8 \mathrm{t}$ (Table 9). Relative emission ranged from 1 to 8 g NMVOC per capita (average 4 g per capita), or $7-25 \mathrm{gt}^{-1}$ of municipal waste (average $13 \mathrm{~g}$ of NMVOC per ton of municipal waste) (Appendices 1 and 2).

Particulate matter. Emission of particulate matter into the air from diesel combustion in Croatian MSW collection systems in 2013, at county level, ranged from 67 to $1500 \mathrm{~kg}$. Total annual emission of PM from MSW collection systems was 8 t. Median value for PM emission at county level was
Diesel consumption for municipal waste collection per capita in Croatian counties in $2013(\mathrm{~kg})$

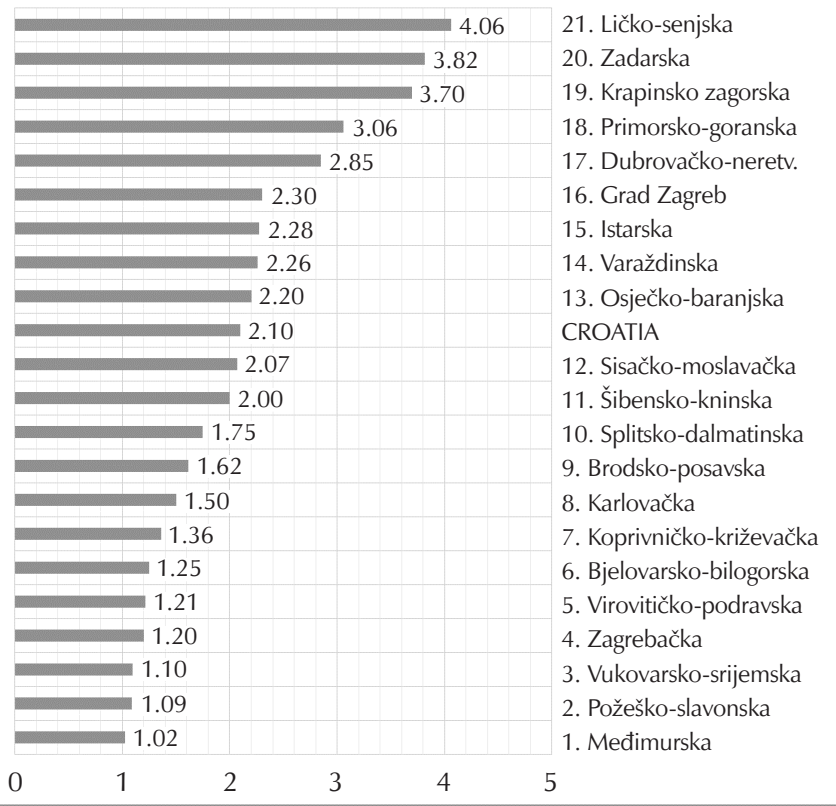

Fig. 4 - Relative diesel consumption per capita for mixed waste collection in Croatian counties in 2013

Slika 4 - Relativna potrošnja dizela u sustavu sakupljanja komunalnog otpada u Hrvatskoj u 2013. godini po stanovniku županije

$240 \mathrm{~kg}$, average $380 \mathrm{~kg}$ (Table 9). Relative emission ranged from 1 to $4 \mathrm{~g}$ PM per capita (average $2 \mathrm{~g}$ PM per capita), or 4-12 $\mathrm{gt}^{-1}$ of municipal waste (average 7 grams of PM per ton of municipal waste) (Appendices 1 and 2).

Black carbon fraction of particulate matter. In the emission of particulate matter, the black carbon fraction ( $\mathrm{f}-\mathrm{BC}$ ) at county level ranged from 36 to $770 \mathrm{~kg}$. Total annual value of black carbon fraction was $4.2 \mathrm{t}$ (more than half of the PM was black carbon fraction). Median value was $130 \mathrm{~kg}$ per county, or on average $200 \mathrm{~kg}$ per county (Table 9). Relative emission ranged from 0 to $2 \mathrm{~g}$ f-BC per capita (average $1 \mathrm{~g}$-BC per capita) or 2-6 $\mathrm{gt}^{-1}$ of municipal waste (average $3 \mathrm{~g}$ of $\mathrm{f}-\mathrm{BC}$ per ton of municipal waste) (Appendices 1 and 2).

Nitrous oxide. Emission of nitrous oxide $\left(\mathrm{N}_{2} \mathrm{O}\right)$ into the air from Croatian MSW collection systems in 2013, at county level, ranged from 3.6 to $79 \mathrm{~kg}$. Total annual emission of $\mathrm{N}_{2} \mathrm{O}$ from MSW collection systems was $435 \mathrm{~kg}$. Median value for $\mathrm{N}_{2} \mathrm{O}$ emission at county level was $13 \mathrm{~kg}$ per county, average $20.7 \mathrm{~kg}$ (Table 9). Relative emission ranged from 39 to $205 \mathrm{mg} \mathrm{N} \mathrm{N}_{2} \mathrm{O}$ per capita (average $102 \mathrm{mg} \mathrm{N} \mathrm{N}_{2} \mathrm{O}$ per capita) or 194-658 $\mathrm{mg} \mathrm{t}^{-1}$ of municipal waste (average $353 \mathrm{mg}$ of $\mathrm{N}_{2} \mathrm{O}$ per ton of municipal waste) (Appendices 1 and 2).

Sulphur dioxide. Emission of sulphur dioxide $\left(\mathrm{SO}_{2}\right)$ into the air from Croatian MSW collection systems in 2013, at county level, ranged from 1.1 to $25 \mathrm{~kg}$. Total annual emission of sulphur from MSW collection systems was $136 \mathrm{~kg}$. 
Table 10 - Diesel related emissions to air from the Croatian municipal solid waste collection in 2013: $\mathrm{SO}_{2}, \mathrm{NH}_{3}, \mathrm{~Pb}, \mathrm{ID}[1,2,3-\mathrm{cd}] \mathrm{P}$, $\mathrm{B}[\mathrm{k}] \mathrm{F}, \mathrm{B}[\mathrm{b}] \mathrm{F}, \mathrm{B}[\mathrm{a}] \mathrm{P}$, and estimated driving distance

Tablica 10 - Emisije u zrak vezane uz potrošnju dizelskog goriva u sustavu sakupljanja komunalnog otpada u Hrvatskoj u 2013. godini: $\mathrm{SO}_{2}, \mathrm{NH}_{3}, \mathrm{~Pb}, \mathrm{ID}[1,2,3-\mathrm{cd}] \mathrm{P}, \mathrm{B}[\mathrm{k}] \mathrm{F}, \mathrm{B}[\mathrm{b}] \mathrm{F}, \mathrm{B}[\mathrm{a}] \mathrm{P}$ te prijeđeni put

\begin{tabular}{|c|c|c|c|c|c|c|c|c|}
\hline Administrative unit & $\mathrm{SO}_{2}$ & $\mathrm{NH}_{3}$ & $\mathrm{~Pb}$ & $\mathrm{ID}[1,2,3-\mathrm{cd}] \mathrm{P}$ & $\mathrm{B}[\mathrm{k}] \mathrm{F}$ & $\mathrm{B}[\mathrm{b}] \mathrm{F}$ & $\mathrm{B}[\mathrm{a}] \mathrm{P}$ & Distance \\
\hline County of & $\mathrm{kg}$ & $\mathrm{kg}$ & g & g & g & g & g & $\mathrm{km}$ \\
\hline Bjelovarsko-bilogorska & 2.08 & 1.69 & 6.78 & 1.03 & 4.48 & 4.01 & 0.66 & 542966.70 \\
\hline Brodsko-posavska & 4.10 & 3.33 & 13.34 & 2.03 & 8.82 & 7.90 & 1.31 & 1068675.85 \\
\hline Dubrovačko-neretvanska & 5.61 & 4.56 & 18.23 & 2.77 & 12.06 & 10.80 & 1.79 & 1460618.02 \\
\hline Grad Zagreb & 28.74 & 23.35 & 93.39 & 14.19 & 61.78 & 55.32 & 9.16 & 7483458.17 \\
\hline Istarska & 7.51 & 6.10 & 24.42 & 3.71 & 16.15 & 14.46 & 2.39 & 1956612.14 \\
\hline Karlovačka & 3.01 & 2.45 & 9.79 & 1.49 & 6.47 & 5.80 & 0.96 & 784153.63 \\
\hline Koprivničko-križevačka & 2.46 & 2.00 & 7.98 & 1.21 & 5.28 & 4.73 & 0.78 & 639610.44 \\
\hline Krapinsko-zagorska & 7.50 & 6.09 & 24.38 & 3.70 & 16.13 & 14.44 & 2.39 & 1953344.56 \\
\hline Ličko-senjska & 3.23 & 2.62 & 10.50 & 1.59 & 6.94 & 6.22 & 1.03 & 841150.56 \\
\hline Međimurska & 1.86 & 1.51 & 6.03 & 0.92 & 3.99 & 3.57 & 0.59 & 483219.02 \\
\hline Osječko-baranjska & 10.60 & 8.62 & 34.46 & 5.24 & 22.80 & 20.41 & 3.38 & 2761368.56 \\
\hline Požeško-slavonska & 1.16 & 0.95 & 3.78 & 0.57 & 2.50 & 2.24 & 0.37 & 302995.92 \\
\hline Primorsko-goranska & 14.50 & 11.78 & 47.12 & 7.16 & 31.17 & 27.91 & 4.62 & 3775393.61 \\
\hline Sisačko-moslavačka & 4.99 & 4.06 & 16.22 & 2.46 & 10.73 & 9.61 & 1.59 & 1299704.64 \\
\hline Splitsko-dalmatinska & 12.72 & 10.33 & 41.34 & 6.28 & 27.35 & 24.48 & 4.05 & 3312338.02 \\
\hline Šibensko-kninska & 3.50 & 2.85 & 11.39 & 1.73 & 7.53 & 6.75 & 1.12 & 912527.38 \\
\hline Varaždinska & 5.88 & 4.78 & 19.12 & 2.91 & 12.65 & 11.33 & 1.88 & 1532349.88 \\
\hline Virovitičko-podravska & 1.64 & 1.33 & 5.33 & 0.81 & 3.52 & 3.15 & 0.52 & 426777.94 \\
\hline Vukovarsko-srijemska & 3.05 & 2.48 & 9.92 & 1.51 & 6.56 & 5.87 & 0.97 & 794564.22 \\
\hline Zadarska & 10.33 & 8.39 & 33.56 & 5.10 & 22.20 & 19.88 & 3.29 & 2689080.38 \\
\hline Zagrebačka & 6.24 & 5.07 & 20.29 & 3.08 & 13.42 & 12.02 & 1.99 & 1625734.33 \\
\hline MIN & 1.16 & 0.95 & 3.78 & 0.57 & 2.50 & 2.24 & 0.37 & 302995.92 \\
\hline MAX & 28.74 & 23.35 & 93.39 & 14.19 & 61.78 & 55.32 & 9.16 & 7483458.17 \\
\hline AVERAGE & 6.70 & 5.44 & 21.78 & 3.31 & 14.41 & 12.90 & 2.14 & 1745078.29 \\
\hline MEDIAN & 4.99 & 4.06 & 16.22 & 2.46 & 10.73 & 9.61 & 1.59 & 1299704.64 \\
\hline CROATIA & 140.72 & 114.34 & 457.35 & 69.48 & 302.55 & 270.89 & 44.86 & 36646643.99 \\
\hline
\end{tabular}

Median value for sulphur emission at county level was $4 \mathrm{~kg}$ per county, average $6.5 \mathrm{~kg}$ (Table 9). Relative emission ranged from 12 to $64 \mathrm{mg} \mathrm{SO}_{2}$ per capita (average $32 \mathrm{mg}$ $\mathrm{SO}_{2}$ per capita) or 61-207 $\mathrm{mg} \mathrm{t}^{-1}$ of municipal waste (average $111 \mathrm{mg} \mathrm{t}^{-1}$ of municipal waste) (Appendices 1 and 2).

Ammonia. Emission of ammonia $\left(\mathrm{NH}_{3}\right)$ into the air from Croatian MSW collection systems in 2013, at county level, ranged from 0.9 to $20 \mathrm{~kg}$. Total annual emission of $\mathrm{NH}_{3}$ from MSW collection systems was $111 \mathrm{~kg}$. Median value for $\mathrm{NH}_{3}$ emission at county level was $3.3 \mathrm{~kg}$ per county, average $5.3 \mathrm{~kg}$ (Table 9). Relative emission ranged from 10 to $52 \mathrm{mg} \mathrm{NH}_{3}$ per capita (average $26 \mathrm{mg} \mathrm{NH}_{3}$ per capita) or 49-168 $\mathrm{mg} \mathrm{t}^{-1}$ of municipal waste (average $90 \mathrm{mg}$ of $\mathrm{NH}_{3}$ per ton of municipal waste) (Appendices 1 and 2).

Lead. Emission of lead $(\mathrm{Pb})$ into the air from Croatian MSW collection systems in 2013, at county level, ranged from 3.8 to $80.4 \mathrm{~kg}$. Total annual emission of lead from MSW collection systems was $443 \mathrm{~kg}$. Median value for lead emission at 
Diesel consumption per mass of collected municipal waste in Croatian counties in 2013 in $\mathrm{kg} / \mathrm{Mg}$

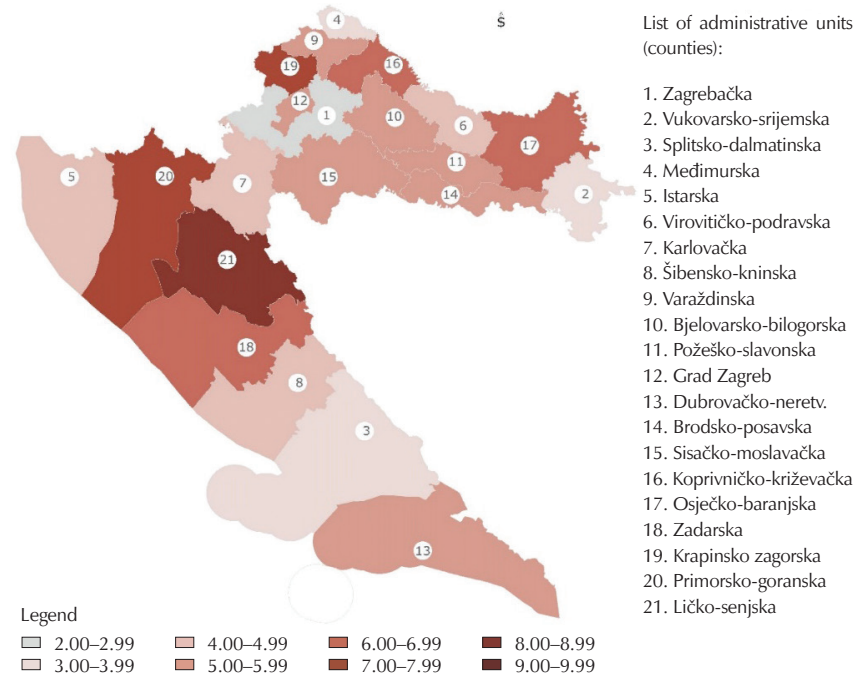

Fig. 6 - Map of relative diesel consumption per mass of collected mixed waste and per capita for mixed waste collection in Croatian counties in 2013

Slika 6 - Karta relativne potrošnja dizela u sustavu sakupljanja komunalnog otpada u Hrvatskoj u 2013. godini po megagramu otpada i po stanovniku županije

county level was $13.2 \mathrm{~kg}$ per county, average $21.1 \mathrm{~kg}$ (Table 9). Relative emission ranged from 40 to $208 \mu \mathrm{g} \mathrm{Pb}$ per capita (average $104 \mu \mathrm{g}$ Pb per capita) or 198-671 $\mu \mathrm{gt}^{-1}$ of municipal waste (average $360 \mu \mathrm{g} \mathrm{t}^{-1}$ of municipal waste) (Appendices 1 and 2).

Indeno[1,2,3-cd]pyrene. Emission of indeno[1,2,3-cd] pyrene into the air from Croatian MSW collection systems in 2013, at county level, ranged from 0.5 to $12 \mathrm{~kg}$. Total annual emission of indeno[1,2,3-cd]pyrene from MSW collection systems was $67 \mathrm{~kg}$. Median value for indeno[1,2,3-cd]pyrene emission at county level was $2 \mathrm{~kg}$ per county, average $3.2 \mathrm{~kg}$ (Table 9). Relative emission ranged from 6 to $32 \mu \mathrm{g}$ indeno[1,2,3-cd]pyrene per capita (average $16 \mu \mathrm{g}$ indeno[1,2,3-cd]pyrene per capita) or 30-102 $\mathrm{gg} \mathrm{t}^{-1}$ of municipal waste (average $55 \mu \mathrm{g}$ of indeno[1,2,3-cd] pyrene per ton of municipal waste) (Appendices 1 and 2).

Benzo[k]fluoranthene. Emission of benzo[k]fluoranthene, $\mathrm{B}[\mathrm{k}] \mathrm{F}$, into the air from Croatian MSW collection systems in 2013, at county level, ranged from 2.5 to $53 \mathrm{~kg}$. Total annual emission of $\mathrm{B}[\mathrm{k}] \mathrm{F}$ from MSW collection systems was $293 \mathrm{~kg}$. Median value for B[k]F emission at county level was $8.8 \mathrm{~kg}$ per county, average $14 \mathrm{~kg}$ (Table 10). Relative emission ranged from 27 to $138 \mu \mathrm{g} \mathrm{B}[\mathrm{k}] \mathrm{F}$ per capita (average $69 \mu \mathrm{g}$ B[k]F per capita) (Table 10), or 131-444 $\mu \mathrm{g} \mathrm{t}^{-1}$ of municipal waste (average $238 \mu \mathrm{gt}^{-1}$ of municipal waste) (Appendices 1 and 2).

Benzo[b]fluoranthene. Emission of benzo[b]fluoranthene, $\mathrm{B}[\mathrm{b}] \mathrm{F}$, into the air from Croatian MSW collection systems in 2013, at county level, ranged from 2.2 to $48 \mathrm{~kg}$. Total annual emission of $\mathrm{B}[\mathrm{b}] \mathrm{F}$ from MSW collection systems was $263 \mathrm{~kg}$. Median value for B[b]F emission at county level was $7.8 \mathrm{~kg}$ per county, average $12.5 \mathrm{~kg}$ (Table 10). Relative emission ranged from 24 to $123 \mu \mathrm{g}$ B[b]F per capita (aver-
Diesel consumption per capita for collection of municipal waste in Croatian counties in 2013 in $\mathrm{kg}$

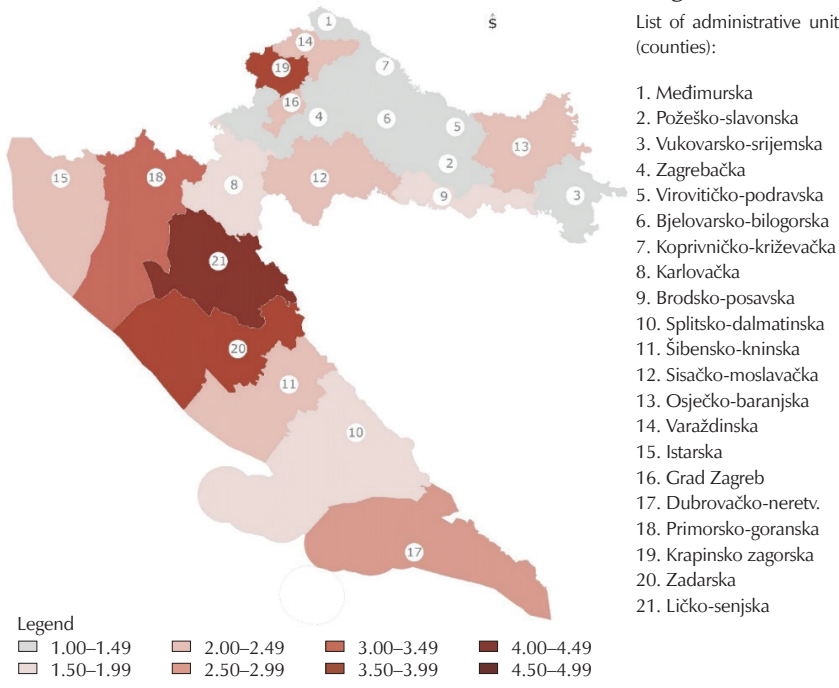

Fig. 7 - Map of relative diesel consumption per capita for mixed waste collection in Croatian counties in 2013

Slika 7 - Karta relativne potrošnja dizela u sustavu sakupljanja komunalnog otpada u Hrvatskoj u 2013. godini po stanovniku županije

Total diesel consumption (in $\mathrm{Mg}$ ) for collection of municipal waste in Croatian counties in 2013

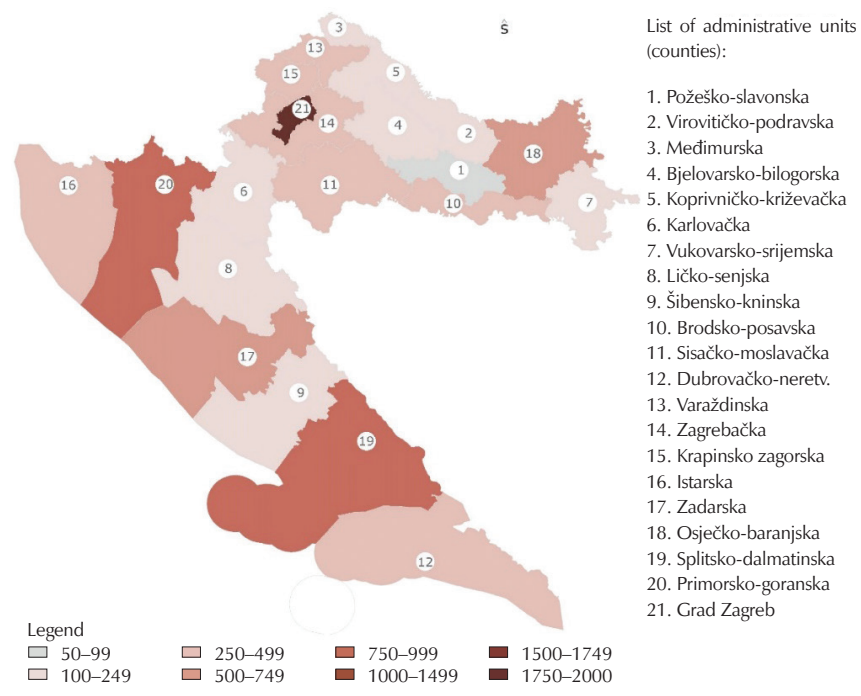

Fig. 8 - Map of total diesel consumption per county related to MSW collection

Slika 8 - Karta potrošnje dizela u sustavu sakupljanja komunalnog otpada

age $62 \mu \mathrm{g}$ B[b]F per capita) or 117-398 $\mu \mathrm{gt}^{-1}$ of municipal

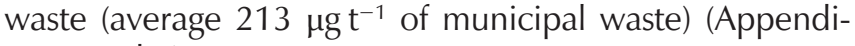
ces 1 and 2).

Benzo[a]pyrene. Emission of benzo[a]pyrene, B[a]P, into the air from Croatian MSW collection systems in 2013, at county level, ranged from 0.4 to $8 \mathrm{~kg}$. Total annual emission of B[a]P from MSW collection systems was $44 \mathrm{~kg}$. Median value for B[a]P emission at county level was $1.3 \mathrm{~kg}$ per 
county, average $2 \mathrm{~kg}$ (Table 10). Relative emission ranged from 4 to $20 \mu \mathrm{g}$ B[a]P per capita (average $10 \mu \mathrm{g}$ B[a]P per capita) or 19-66 $\mu \mathrm{g} \mathrm{t}^{-1}$ of municipal waste (average

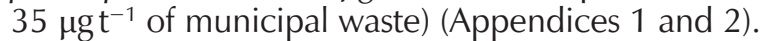

Transport distance. Total annual transport distance in Croatian MSW collection systems in 2013 was estimated at 36.7 million $\mathrm{km}$. From county to county, the distance varied between 303 thousand $\mathrm{km}$ and 7.5 million $\mathrm{km}$; median value was 1.3 million $\mathrm{km}$ (Table 10). Relative transport distance ranged from 11.3 to $36.8 \mathrm{~km} \mathrm{t}^{-1}$ of collected waste in a county, or $4.3-16.9 \mathrm{~km}$ per inhabitant of the county. Median values were $22 \mathrm{~km} \mathrm{t}^{-1}$ or $8.4 \mathrm{~km}$ per inhabitant of the county. The Croatian average was $22.5 \mathrm{~km} \mathrm{t}^{-1}$ or $8.7 \mathrm{~km}$ per capita. (Appendices 1 and 2)

\subsection{Discussion}

The emission results calculated with Tier 1 emission factors for diesel fuel are more comprehensive than the results in introductory literature. Tier 1 emission factors are, on the environmental side, showing somewhat higher emissions than Tier 2 and 3, which are based not only on the type of vehicle and quantity of the consumed fuel, but also include kilometres driven and average fuel consumption, as well as load and other elements. ${ }^{21}$

In different studies, emission of $\mathrm{CO}_{2}$ ranged from 1.1 to $703 \mathrm{~kg}$ of $\mathrm{CO}_{2}$ per ton of waste:

- 7-9 $\mathrm{kg}$ of fossil $\mathrm{CO}_{2}$ equivalent per ton for mixed waste transported to a landfill ${ }^{16,17}$

- 8-15 kg t $\mathrm{t}^{-1}$ for various waste streams $\mathrm{s}^{16,17}$

- 1.1-7.6 $\mathrm{kg} \mathrm{t}^{-1}$ for Dresden ${ }^{18}$ which is per inhabitant number similar to the city of Zagreb

- 11.9-90 $\mathrm{kg} \mathrm{t}^{-1}$ for different truck types ${ }^{19}$

- 12 to $703 \mathrm{~kg}$ of $\mathrm{CO}_{2}$ (GWP) per ton of different waste streams and type of treatment included ${ }^{20}$

As shown in the previous chapter (Table 10), values for Croatian municipal solid waste collection system range from 8.6 to $28.1 \mathrm{~kg}$ of $\mathrm{CO}_{2}$ per ton, and average emission is $17 \mathrm{~kg}$ of $\mathrm{CO}_{2}$ per ton of collected mixed waste. These results place Croatia far from the emission average of 7-9 kg of $\mathrm{CO}_{2}$ per ton of collected mixed waste. Some counties, however, appear to be closer to standard $\mathrm{CO}_{2}$ (eq.) emission factor per ton of waste, for example administrative units Zagrebačka (8.6 kg of $\mathrm{CO}_{2}$ per ton), Vukovarsko-srijemska (11.5 $\left.\mathrm{kg} \mathrm{t}^{-1}\right)$, Splitsko-dalmatinska $\left(11.7 \mathrm{~kg} \mathrm{t}^{-1}\right)$ etc., as given previously in Table 9. The results should be observed with data representativeness in perspective (shown in Table 4), which for the respective counties is $8.61 \%$, $49.75 \%$ and $52.82 \%$. Additionally, representativeness for the waste stream "mixed waste" is $76.60 \%, 97.29 \%$ and $96.29 \%$. The data with higher representativeness give results with higher reliability.

The AEA study ${ }^{16}$ shows higher $\mathrm{CO}_{2}$ emission per mass of transported waste for separated waste fractions. This sug- gests that, when the collection rates of separate waste streams increase, the $\mathrm{CO}_{2}$ burden will increase as well, due to their differences in mass per volume (load of trucks) and transport distance to recycling facilities.

The results for the city of Zagreb are far from the results for the city of Dresden, however, the main collector of MSW from Zagreb city delivered no data, so the reliability of the estimate for the city of Zagreb is uncertain. Therefore, it was represented by Croatian average values.

Comparison of Croatian $\mathrm{CO}_{2}$ emission with the results of the study for different truck types, ${ }^{19}$ show that average technology in current use in Croatia have decent fuel efficiency (at least compared to USA technology). However, the efforts to lower both the quantity of waste and the $\mathrm{CO}_{2}$ emission per ton of waste should be continued. That would decrease the impact on human health and ecosystems caused by waste collection, as well as the operating costs of the waste collection companies. Investment to switch to cleaner technologies could however be significant, and should be considered at strategic level.

\section{Conclusion}

This article presents the fuel consumption and fuel related airborne emissions from collection of mixed municipal waste. The data is relevant for Croatia, 2013, and the mixed waste stream. The data is presented in both absolute and relative values (relative to the number of inhabitants and mass of collected waste) per county and for the whole country. Annual consumption of diesel for collection of mixed municipal waste in 2013, at county level, ranged from 87 thousand litres to 2.2 million litres, on average 504 thousand litres per county. Relative consumption of diesel was $6.4 \mathrm{It}^{-1}$ of collected waste (ranging from 3.2 to 10.6 I), or 2.5 I per capita (1.2-4.9 I). Total consumption of diesel for collection of mixed municipal waste in Croatia in 2013 was estimated at 10.6 million litres.

Estimated emission of carbon dioxide into the air from diesel combustion ranged from 231 to $5711 \mathrm{t}$. Total annual emission of $\mathrm{CO}_{2}$ was close to $28000 \mathrm{t}$, on average $1331 \mathrm{t}$ per county. Relative emission ranged from 3.3 to $13 \mathrm{~kg} \mathrm{CO}_{2}$ per capita (average $6.6 \mathrm{~kg}$ per capita), or $8.6-28.1 \mathrm{~kg} \mathrm{t}^{-1}$ of mixed municipal waste (average $17 \mathrm{~kg}$ of $\mathrm{CO}_{2}$ per ton). The average values of $\mathrm{CO}_{2}$ emission from waste collection that should also be the target values are 7-9 $\mathrm{kg}$ for mixed waste, and $8-15 \mathrm{~kg} \mathrm{CO}_{2}$ for separate waste streams. Together with the aim to lower the $\mathrm{CO}_{2}$ emission per ton of waste, efforts to lower the quantity of waste and awareness raising should be continued. That would decrease the impact on human health and ecosystems caused by waste collection, as well as the operating costs of the waste collection companies. Investment to switch to cleaner technologies could however be significant, and should be considered at strategic level, and backed up by life cycle studies of various waste management options and their overall emissions and impacts. 


\section{ACKNOWLEDGEMENTS}

This article is not funded by any institution or company whatsoever; therefore, the author declares no conflict of interest. The author expresses gratitude to anonymous reviewers whose valuable comments incited major revision of the data and improvement to presentation of the topic.

\section{List of abbreviations and symbols Popis kratica i simbola}

\begin{tabular}{|c|c|}
\hline$A D$ & $\begin{array}{l}\text { - anaerobic digestion } \\
\text { - anaerobna digestija }\end{array}$ \\
\hline $\mathrm{B}[\mathrm{a}] \mathrm{P}$ & $\begin{array}{l}\text { - benzo[a]pyrene } \\
\text { - benzo[a]piren }\end{array}$ \\
\hline $\mathrm{B}[\mathrm{b}] \mathrm{F}$ & $\begin{array}{l}\text { - benzo[b]fluoranthene } \\
\text { - benzo[b]fluoranten }\end{array}$ \\
\hline $\mathrm{B}[\mathrm{k}] \mathrm{F}$ & $\begin{array}{l}\text { - benzo[k]fluoranthene } \\
\text { - benzo[k]fluoranten }\end{array}$ \\
\hline $\mathrm{CO}$ & $\begin{array}{l}\text { - carbon monoxide } \\
\text { - ugljikov monoksid }\end{array}$ \\
\hline $\mathrm{CO}_{2}$ & $\begin{array}{l}\text { - carbon dioxide } \\
\text { - ugljikov dioksid }\end{array}$ \\
\hline$f-B C$ & $\begin{array}{l}\text { - black carbon fraction of particulate matter } \\
\text { - frakcije čestica crnog ugljika }\end{array}$ \\
\hline $\mathrm{HC}$ & $\begin{array}{l}\text { - hydrocarbons } \\
\text { - hidrokarbonati }\end{array}$ \\
\hline LCA & $\begin{array}{l}\text { - life cycle assessment } \\
\text { - procjena životnog ciklusa }\end{array}$ \\
\hline MSW & $\begin{array}{l}\text { - municipal solid waste } \\
\text { - komunalni kruti otpad }\end{array}$ \\
\hline MRF & $\begin{array}{l}\text { - materials recovery facility } \\
\text { - oporavak materijala }\end{array}$ \\
\hline $\mathrm{N}_{2} \mathrm{O}$ & $\begin{array}{l}\text { - nitrous oxide } \\
\text { - dušikov(I) oksid }\end{array}$ \\
\hline $\mathrm{NH}_{3}$ & $\begin{array}{l}\text { - ammonia } \\
\text { - amonijak }\end{array}$ \\
\hline NMVC & $\begin{array}{l}\text { - non-methane volatile organic compounds } \\
\text { - nemetanski isparljivi organski spojevi }\end{array}$ \\
\hline $\mathrm{NO}_{x}$ & $\begin{array}{l}\text { - nitrogen oxides } \\
\text { - dušikovi oksidi }\end{array}$ \\
\hline PM & $\begin{array}{l}\text { - particulate matter } \\
\text { - čestice }\end{array}$ \\
\hline RTS & $\begin{array}{l}\text { - refuse transfer station } \\
\text { - pretovarna stanica }\end{array}$ \\
\hline WM & $\begin{array}{l}\text { - waste management } \\
\text { - upravljanje otpadom }\end{array}$ \\
\hline
\end{tabular}

\section{References Literatura}

1. A. Grbeš, I. Ljubić, Ž. Veinović, Fuel related $\mathrm{CO}_{2}$ emission of municipal solid waste collection system in Croatia, in Z. Katančić, N. Koprivanac, A. Lončarić Božić, H. Kušić, Z. Hrnjak-Murgić (Eds.), Conference Proceedings of SEM2016, Zagreb, University of Zagreb, Faculty of Chemical Engineering and Technology, 2016, pp. 61-66.

2. H. J. Bjarnadóttir, G. B. Friðriksson, T. Johnsen, H. Sletsen, Guidelines for the use of LCA in the waste management sector, Nordtest Report TR 517, Approved 2002-11, URL: http://infohouse.p2ric.org/ref/37/36469.pdf (4/2015).

3. G. Finnveden, T. Ekvall, Life-cycle assessment as a decision-support tool - the case of recycling versus incineration of paper, Resour. Conserv. Recy. 24 (1998) 235-256, doi: https://doi.org/10.1016/S0921-3449(98)00039-1.

4. G. Finnveden, On the Possibilities of Life-Cycle Assessment - Development of Methodology and Review of Case Studies, Doctoral thesis in Natural Resources Management, Department of Systems Ecology, Stockholm University, 1998 (4/2015)

5. R. Clift, A. Doig, G. Finnveden, Application of Life Cycle Assessment to Integrated Solid Waste Management. Part 1 Methodology, Process Saf. Environ. Prot. Trans. Inst. Chem. Eng. Part B. 78 (4) (2000) 279-287, doi: http://dx.doi. org/10.1205/095758200530790.

6. T. Ekvall, G. Finnveden, The Application of Life Cycle Assessment to Integrated Solid Waste Management. Part 2 - Perspectives on Energy and Material Recovery from Paper, Process Saf. Environ. Prot. Trans. Inst. Chem. Eng. Part B. 78 (4) (2000) 288-294, doi: https://doi. org/10.1205/095758200530808.

7. O. Eriksson, B. Frostell, A. Björklund, G. Assefa, J. O. Sundqvist, J. Granath, M. C. Reich, A. Baky, L. Thyselius, Energy Recovery and Material and Nutrient Recycling from a System Perspective, A paper presented at the international workshop "System Analyses of Integrated Waste Management" in Johannesberg Castle, Sweden, 2-3 April 2001.

8. G. A. Blengini, G. Genon, M. Fantoni, LCA of integrated municipal solid waste management systems: Case studies of Torino and Cuneo (Italy) Proceedings Sardinia 2011, Thirteenth International Waste Management and Landfill Symposium S. Margherita di Pula, Cagliari, Italy; 3-7 October 2011, CISA Publisher, Italy, URL: http://www.sardiniasymposium.it/public/images/pdf/Blengini.pdf (4/2015)

9. L. Rigamonti, M. Grosso, M Giugliano, Life cycle assessment of sub-units composing a MSW management system, J. Clean. Prod. 18 (2010) 1652-1662, doi: https://doi.org/10.1016/j. jclepro.2010.06.029.

10. G. Finnveden, Methodological aspects of life cycle assessment of integrated solid waste management systems, Resour. Conserv. Recy. 26 (1999) 173-187, doi: https://doi. org/10.1016/S0921-3449(99)00005-1.

11. J. Kirkeby, Modelling of life cycle assessment of solid waste management systems and technologies, PhD Thesis, Insti- 
tute of Environment and Resources, Technical University of Denmark (2005), URL: http://orbit.dtu.dk/fedora/objects/orbit:86045/datastreams/file_5584219/content (4/2015).

12. E. Williams, Environmental life cycle assessment and municipal solid waste management, Encyclopedia of Life Support Systems (EOLSS), Human Settlement Development - Vol. III. (2007), URL: http://www.eolss.net/sample-chapters/c14/E118-06-07.pdf (4/2015).

13. S. S. Naghibzadeh, N. Khorasani, J. Yousefi, B. S. Mousavi, Z. Badehian, Life Cycle Assessment of Municipal Waste Management System (Case Study: Karaj, Iran), J. Appl. Sci. Environ. Manag. 18 (4) (2014) 559-565, url: http://www.bioline. org.br/pdf?ja14074.

14. I. Ljubić, Analysis of the municipal solid waste system in Croatia: Key indicators and cartographic view, Master thesis, University of Zagreb, Faculty of Mining, Geology and Petroleum Engineering, Zagreb (2015).

15. AZO, Agencija za zaštitu okoliša, Izvješće o komunalnom otpadu za 2013. godinu, (2014) [Croatian Environment Agency (2014): Report on Municipal Waste for 2013], URL: http://www.azo.hr/lzvjesca25 (4/2015).

16. AEA, Waste Management Options and Climate Change (2001), URL: http://ec.europa.eu/environment/waste/studies/pdf/climate_change.pdf (6. 2. 2017.)
17. D. Teichmann, Ch. Schempp, Calculation of GHG Emissions of Waste management Projects, JASPERS (Joint Assistance to Support Projects in European Regions) Knowledge Economy and Energy Division, (2013), URL: http://www.jaspersnetwork.org/ (3. 2. 2017.).

18. J. Winkler, B. Bilitewski, Comparative evaluation of life cycle assessment models for solid waste management, Waste Manag. 27 (2007) 1021-1031, doi: https://doi.org/10.1016/j. wasman.2007.02.023.

19. L. D. Hauser, Evaluating the Air Emissions from Solid Waste Refuse Trucks, Civil Engineering Theses, Dissertations, and Student Research, (2015) Paper 79, University of Nebraska-Lincoln, URL: http://digitalcommons.unl.edu/civilengdiss/79 (7. 2. 2017.)

20. S. Salhofer, F. Schneider, G. Obersteiner, The ecological relevance of transport in waste disposal systems in Western Europe, Waste Manag. 27 (2007) S47-S57, doi: https://doi. org/10.1016/j.wasman.2007.02.025.

21. L. Ntziachristos, Z. Samaras, European Environmental Agency, EEA: EMEP/EEA air pollutant emission inventory guidebook 2016- Update Dec. 2016. 1.A.3.b.i, 1.A.3.b.ii, 1.A.3.b.iii, 1.A.3.b.iv. Passenger cars, light commercial trucks, heavy-duty vehicles including buses and motor cycles, URL: http://www.eea.europa.eu/publications/emepeea-guidebook-2016 (15. 2. 2017.). 


\section{Appendices}

Appendix 1 - Diesel related emissions into air from the Croatian municipal solid waste collection in 2013 per mass of collected waste Dodatak 1 - Emisije u zrak vezane uz potrošnju dizelskog goriva u sustavu sakupljanja komunalnog otpada u Hrvatskoj u 2013. godini po jedinici mase sakupljenog otpada

\begin{tabular}{|c|c|c|c|c|c|c|c|c|c|c|c|c|c|c|c|c|c|}
\hline $\begin{array}{l}\text { Administrative } \\
\text { unit (county) }\end{array}$ & Diesel & $\mathrm{CO}_{2}$ & $\mathrm{NO}_{x}$ & $\mathrm{CO}$ & 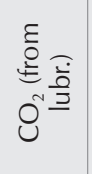 & $\sum_{Z}^{\bigcup}$ & PM & $f-B C$ & $\mathrm{~N}_{2} \mathrm{O}$ & $\mathrm{SO}_{2}$ & $\mathrm{NH}_{3}$ & $\mathrm{~Pb}$ & 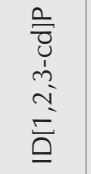 & $\mathrm{B}[\mathrm{k}] \mathrm{F}$ & $\mathrm{B}[\mathrm{b}] \mathrm{F}$ & $\mathrm{B}[\mathrm{a}] \mathrm{P}$ & 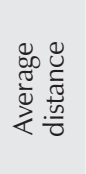 \\
\hline & kg & kg & g & g & g & g & g & g & g & $\mathrm{mg}$ & g & $\mu \mathrm{g}$ & $\mu \mathrm{g}$ & $\mu g$ & $\mu g$ & $\mu g$ & $\mathrm{~km}$ \\
\hline $\begin{array}{l}\text { Bjelovarsko- } \\
\text { bilogorska }\end{array}$ & 1.25 & 3.97 & 41.65 & 9.46 & 3.17 & 2.40 & 1.17 & 0.62 & 0.06 & 19.97 & 0.02 & 64.90 & 9.86 & 42.93 & 38.44 & 6.36 & 5.20 \\
\hline $\begin{array}{l}\text { Brodsko- } \\
\text { posavska }\end{array}$ & 1.62 & 5.14 & 53.98 & 12.26 & 4.11 & 3.11 & 1.52 & 0.81 & 0.08 & 25.88 & 0.02 & 84.11 & 12.78 & 55.64 & 49.82 & 8.25 & 6.74 \\
\hline $\begin{array}{l}\text { Dubrovačko- } \\
\text { neretvanska }\end{array}$ & 2.85 & 9.06 & 95.07 & 21.60 & 7.24 & 5.47 & 2.68 & 1.42 & 0.15 & 45.58 & 0.04 & 148.15 & 22.51 & 98.01 & 87.75 & 14.53 & 11.87 \\
\hline Grad Zagreb & 2.30 & 7.32 & 76.84 & 17.45 & 5.85 & 4.42 & 2.16 & 1.15 & 0.12 & 36.84 & 0.03 & 119.74 & 18.19 & 79.21 & 70.92 & 11.74 & 9.59 \\
\hline Istarska & 2.28 & 7.24 & 75.96 & 17.26 & 5.78 & 4.37 & 2.14 & 1.13 & 0.12 & 36.42 & 0.03 & 118.37 & 17.98 & 78.31 & 70.11 & 11.61 & 9.48 \\
\hline Karlovačka & 1.50 & 4.77 & 50.03 & 11.36 & 3.81 & 2.88 & 1.41 & 0.75 & 0.08 & 23.99 & 0.02 & 77.96 & 11.84 & 51.57 & 46.18 & 7.65 & 6.25 \\
\hline $\begin{array}{l}\text { Koprivničko- } \\
\text { križevačka }\end{array}$ & 1.36 & 4.33 & 45.42 & 10.32 & 3.46 & 2.61 & 1.28 & 0.68 & 0.07 & 21.78 & 0.02 & 70.78 & 10.75 & 46.82 & 41.92 & 6.94 & 5.67 \\
\hline $\begin{array}{l}\text { Krapinsko- } \\
\text { zagorska }\end{array}$ & 3.70 & 11.76 & 123.45 & 28.04 & 9.40 & 7.10 & 3.48 & 1.84 & 0.19 & 59.19 & 0.05 & 192.37 & 29.23 & 127.26 & 113.94 & 18.87 & 15.41 \\
\hline Ličko-senjska & 4.06 & 12.92 & 135.55 & 30.79 & 10.32 & 7.80 & 3.82 & 2.02 & 0.21 & 64.99 & 0.05 & 211.22 & 32.09 & 139.73 & 125.11 & 20.72 & 16.92 \\
\hline Međimurska & 1.02 & 3.26 & 34.19 & 7.77 & 2.60 & 1.97 & 0.96 & 0.51 & 0.05 & 16.39 & 0.01 & 53.28 & 8.09 & 35.24 & 31.56 & 5.23 & 4.27 \\
\hline $\begin{array}{l}\text { Osječko- } \\
\text { baranjska }\end{array}$ & 2.20 & 7.00 & 73.48 & 16.69 & 5.59 & 4.23 & 2.07 & 1.10 & 0.11 & 35.23 & 0.03 & 114.51 & 17.40 & 75.75 & 67.82 & 11.23 & 9.18 \\
\hline $\begin{array}{l}\text { Požeško- } \\
\text { slavonska }\end{array}$ & 1.09 & 3.45 & 36.22 & 8.23 & 2.76 & 2.08 & 1.02 & 0.54 & 0.06 & 17.37 & 0.01 & 56.44 & 8.57 & 37.34 & 33.43 & 5.54 & 4.52 \\
\hline $\begin{array}{l}\text { Primo } \\
\text { goran }\end{array}$ & 3.06 & 9.73 & 102.15 & 23.20 & 7.78 & 5.88 & 2.88 & 1.53 & 0.16 & 48.98 & 0.04 & 159.19 & 24.18 & 105.31 & 94.29 & 15.61 & 12.76 \\
\hline $\begin{array}{l}\text { Sisačko- } \\
\text { moslavačka }\end{array}$ & 2.07 & 6.58 & 69.07 & 15.69 & 5.26 & 3.97 & 1.95 & 1.03 & 0.11 & 33.12 & 0.03 & 107.64 & 16.35 & 71.21 & 63.75 & 10.56 & 8.62 \\
\hline $\begin{array}{l}\text { Splitsko- } \\
\text { dalmatinska }\end{array}$ & 1.75 & 5.56 & 58.31 & 13.25 & 4.44 & 3.36 & 1.64 & 87 & 0.09 & 27.96 & .02 & 90.87 & 13.80 & 60.11 & 53.82 & 8.91 & 7.28 \\
\hline $\begin{array}{l}\text { Šibensko- } \\
\text { kninska }\end{array}$ & 2.00 & 6.37 & 66.84 & 15.18 & 5.09 & 3.85 & 1.88 & 1.00 & 0.10 & 32.05 & 0.03 & 104.15 & 15.82 & 68.90 & 61.69 & 10.21 & 8.35 \\
\hline Varaždinska & 2.26 & 19 & 75.40 & 17.13 & 5.74 & 4.34 & 2.12 & 1.13 & 0.12 & 36.15 & 0.03 & 117.50 & 17.85 & 77.73 & 69.59 & 11.52 & 9.41 \\
\hline $\begin{array}{l}\text { Virovitičko- } \\
\text { podravska }\end{array}$ & 1.21 & 3.86 & 40.47 & 9.19 & 3.08 & 2.33 & 1.14 & 0.60 & 0.06 & 19.41 & 0.02 & 63.07 & 9.58 & 41.72 & 37.36 & 6.19 & 5.05 \\
\hline $\begin{array}{l}\text { Vukovarsko- } \\
\text { srijemska }\end{array}$ & 1.10 & 3.49 & 36.62 & 8.32 & 2.79 & 2.11 & 1.03 & 0.55 & 0.06 & 17.56 & 0.01 & 57.07 & 8.67 & 37.75 & 33.80 & 5.60 & 4.57 \\
\hline Zadarska & 3.82 & 12.13 & 127.31 & 28.92 & 9.69 & 7.32 & 3.59 & 1.90 & 0.19 & 61.04 & 0.05 & 198.38 & 30.14 & 131.24 & 117.50 & 19.46 & 15.90 \\
\hline Zagrebačka & 1.20 & 3.82 & 40.03 & 9.09 & 3.05 & 2.30 & 1.13 & 0.60 & 0.06 & 19.20 & 0.02 & 62.38 & 9.48 & 41.27 & 36.95 & 6.12 & 5.00 \\
\hline MIN & 1.02 & 3.26 & 34.19 & 7.77 & 2.60 & 1.97 & 0.96 & 0.51 & 0.05 & 16.39 & 0.01 & 53.28 & 8.09 & 35.24 & 31.56 & 5.23 & 4.27 \\
\hline MAX & 4.06 & 12.92 & 135.55 & 30.79 & 10.32 & 7.80 & 3.82 & 2.02 & 0.21 & 64.99 & 0.05 & 211.22 & 32.09 & 139.73 & 125.11 & 20.72 & 16.92 \\
\hline AVERAGE & 2.08 & 6.62 & 69.43 & 15.77 & 5.28 & 3.99 & 1.96 & 1.04 & 0.11 & 33.29 & 0.03 & 108.19 & 16.44 & 71.57 & 64.08 & 10.61 & 8.67 \\
\hline MEDIAN & 2.00 & 6.37 & 66.84 & 15.18 & 5.09 & 3.85 & 1.88 & 1.00 & 0.10 & 32.05 & 0.03 & 104.15 & 15.82 & 68.90 & 61.69 & 10.21 & 8.35 \\
\hline CROATIA & 2.10 & 6.67 & 69.97 & 15.89 & 5.33 & 4.03 & 1.97 & 1.04 & 0.11 & 33.55 & 0.03 & 109.03 & 16.56 & 72.13 & 64.58 & 10.69 & 8.74 \\
\hline
\end{tabular}


Appendix 2 - Diesel related emissions into air from the Croatian municipal solid waste collection in 2013 per capita

Dodatak 2 - Emisije u zrak vezane uz potrošnju dizelskog goriva u sustavu sakupljanja komunalnog otpada u Hrvatskoj u 2013. godini per capita

\begin{tabular}{|c|c|c|c|c|c|c|c|c|c|c|c|c|c|c|c|c|c|}
\hline $\begin{array}{l}\text { Administrative } \\
\text { unit (county) }\end{array}$ & Diesel & $\mathrm{CO}_{2}$ & $\mathrm{NO}_{x}$ & $\mathrm{CO}$ & $\underbrace{\frac{\varepsilon}{0}}_{0^{N}}$ & $\sum_{Z}^{\bigcup}$ & PM & $f-B C$ & $\mathrm{~N}_{2} \mathrm{O}$ & $\mathrm{SO}_{2}$ & $\mathrm{NH}_{3}$ & $\mathrm{~Pb}$ & 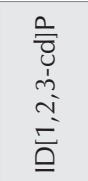 & $\mathrm{B}[\mathrm{k}] \mathrm{F}$ & $\mathrm{B}[\mathrm{b}] \mathrm{F}$ & $\mathrm{B}[\mathrm{a}] \mathrm{P}$ & 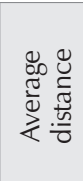 \\
\hline & $\mathrm{kg}$ & $\mathrm{kg}$ & g & g & g & g & $\mathrm{g}$ & g & g & $\mathrm{mg}$ & g & $\mu g$ & $\mu g$ & $\mathrm{Mg}$ & $\mu g$ & $\mu g$ & $\mathrm{~km}$ \\
\hline $\begin{array}{l}\text { Bjelovarsko- } \\
\text { bilogorska }\end{array}$ & 1.25 & 3.97 & 41.65 & 9.46 & 3.17 & 2.40 & 1.17 & 0.62 & 0.06 & 19.97 & 0.02 & 64.90 & 9.86 & 42.93 & 38.44 & 6.36 & 5.20 \\
\hline $\begin{array}{l}\text { Brodsko- } \\
\text { posavska }\end{array}$ & 1.62 & 5.14 & 53.98 & 12.26 & 4.11 & 3.11 & 1.52 & 0.81 & 0.08 & 25.88 & 0.02 & 84.11 & 12.78 & 55.64 & 49.82 & 8.25 & 6.74 \\
\hline $\begin{array}{l}\text { Dubrovačko- } \\
\text { neretvanska }\end{array}$ & 2.85 & 9.06 & 95.07 & 21.60 & 7.24 & 5.47 & 2.68 & 1.42 & 0.15 & 45.58 & 0.04 & 148.15 & 22.51 & 98.01 & 87.75 & 14.53 & 11.87 \\
\hline Grad Zagreb & 2.30 & 7.32 & 76.84 & 17.45 & 5.85 & 4.42 & 2.16 & 1.15 & 0.12 & 36.84 & 0.03 & 119.74 & 18.19 & 79.21 & 70.92 & 11.74 & 9.59 \\
\hline Istarska & 2.28 & 7.24 & 75.96 & 17.26 & 5.78 & 4.37 & 2.14 & 1.13 & 0.12 & 36.42 & 0.03 & 118.37 & 17.98 & 78.31 & 70.11 & 11.61 & 9.48 \\
\hline Karlovačka & 1.50 & 4.77 & 50.03 & 11.36 & 3.81 & 2.88 & 1.41 & 0.75 & 0.08 & 23.99 & 0.02 & 77.96 & 11.84 & 51.57 & 46.18 & 7.65 & 6.25 \\
\hline $\begin{array}{l}\text { Koprivničko- } \\
\text { križevačka }\end{array}$ & 1.36 & 4.33 & 45.42 & 10.32 & 3.46 & 2.61 & 1.28 & 0.68 & 0.07 & 21.78 & 0.02 & 70.78 & 10.75 & 46.82 & 41.92 & 6.94 & 5.67 \\
\hline $\begin{array}{l}\text { Krapinsko- } \\
\text { zagorska }\end{array}$ & 3.70 & 11.76 & 123.45 & 28.04 & 9.40 & 7.10 & 3.48 & 1.84 & 0.19 & 59.19 & 0.05 & 192.37 & 29.23 & 127.26 & 113.94 & 18.87 & 15.41 \\
\hline Ličko-senjska & 4.06 & 12.92 & 135.55 & 30.79 & 10.32 & 7.80 & 3.82 & 2.02 & 0.21 & 64.99 & 0.05 & 211.22 & 32.09 & 139.73 & 125.11 & 20.72 & 16.92 \\
\hline Međimurska & 1.02 & 3.26 & 34.19 & 7.77 & 2.60 & 1.97 & 0.96 & 0.51 & 0.05 & 16.39 & 0.01 & 53.28 & 8.09 & 35.24 & 31.56 & 5.23 & 4.27 \\
\hline $\begin{array}{l}\text { Osječko- } \\
\text { baranjska }\end{array}$ & 2.20 & 7.00 & 73.48 & 16.69 & 5.59 & 4.23 & 2.07 & 1.10 & 0.11 & 35.23 & 0.03 & 114.51 & 17.40 & 75.75 & 67.82 & 11.23 & 9.18 \\
\hline $\begin{array}{l}\text { Požeško- } \\
\text { slavonska }\end{array}$ & 1.09 & 3.45 & 36.22 & 8.23 & 2.76 & 2.08 & 1.02 & 0.54 & 0.06 & 17.37 & 0.01 & 56.44 & 8.57 & 37.34 & 33.43 & 5.54 & 4.52 \\
\hline $\begin{array}{l}\text { Primorsko- } \\
\text { goranska }\end{array}$ & 3.06 & 9.73 & 102.15 & 23.20 & 7.78 & 5.88 & 2.88 & 1.53 & 0.16 & 48.98 & 0.04 & 159.19 & 24.18 & 105.31 & 94.29 & 15.61 & 12.76 \\
\hline $\begin{array}{l}\text { Sisačko- } \\
\text { moslavačka }\end{array}$ & 2.07 & 6.58 & 69.07 & 15.69 & 5.26 & 3.97 & 1.95 & 1.03 & 0.11 & 33.12 & 0.03 & 107.64 & 16.35 & 71.21 & 63.75 & 10.56 & 8.62 \\
\hline $\begin{array}{l}\text { Splitsko- } \\
\text { dalmatinska }\end{array}$ & 1.75 & 5.56 & 58.31 & 13.25 & 4.44 & 3.36 & 1.64 & 0.87 & 0.09 & 27.96 & 0.02 & 90.87 & 13.80 & 60.11 & 53.82 & 8.91 & 7.28 \\
\hline $\begin{array}{l}\text { Šibensko- } \\
\text { kninska }\end{array}$ & 2.00 & 6.37 & 66.84 & 15.18 & 5.09 & 3.85 & 1.88 & 1.00 & 0.10 & 32.05 & 0.03 & 104.15 & 15.82 & 68.90 & 61.69 & 10.21 & 8.35 \\
\hline Varaždinska & 2.26 & 7.19 & 75.40 & 17.13 & 5.74 & 4.34 & 2.12 & 1.13 & 0.12 & 36.15 & 0.03 & 117.50 & 17.85 & 77.73 & 69.59 & 11.52 & 9.41 \\
\hline $\begin{array}{l}\text { Virovitičko- } \\
\text { podravska }\end{array}$ & 1.21 & 3.86 & 40.47 & 9.19 & 3.08 & 2.33 & 1.14 & 0.60 & 0.06 & 19.41 & 0.02 & 63.07 & 9.58 & 41.72 & 37.36 & 6.19 & 5.05 \\
\hline $\begin{array}{l}\text { Vukovarsko- } \\
\text { srijemska }\end{array}$ & 1.10 & 3.49 & 36.62 & 8.32 & 2.79 & 2.11 & 1.03 & 0.55 & 0.06 & 17.56 & 0.01 & 57.07 & 8.67 & 37.75 & 33.80 & 5.60 & 4.57 \\
\hline Zadarska & 3.82 & 12.13 & 127.31 & 28.92 & 9.69 & 7.32 & 3.59 & 1.90 & 0.19 & 61.04 & 0.05 & 198.38 & 30.14 & 131.24 & 117.50 & 19.46 & 15.90 \\
\hline Zagrebačka & 1.20 & 3.82 & 40.03 & 9.09 & 3.05 & 2.30 & 1.13 & 0.60 & 0.06 & 19.20 & 0.02 & 62.38 & 9.48 & 41.27 & 36.95 & 6.12 & 5.00 \\
\hline MIN & 1.02 & 3.26 & 34.19 & 7.77 & 2.60 & 1.97 & 0.96 & 0.51 & 0.05 & 16.39 & 0.01 & 53.28 & 8.09 & 35.24 & 31.56 & 5.23 & 4.27 \\
\hline MAX & 4.06 & 12.92 & 135.55 & 30.79 & 10.32 & 7.80 & 3.82 & 2.02 & 0.21 & 64.99 & 0.05 & 211.22 & 32.09 & 139.73 & 125.11 & 20.72 & 16.92 \\
\hline AVERAGE & 2.08 & 6.62 & 69.43 & 15.77 & 5.28 & 3.99 & 1.96 & 1.04 & 0.11 & 33.29 & 0.03 & 108.19 & 16.44 & 71.57 & 64.08 & 10.61 & 8.67 \\
\hline MEDIAN & 2.00 & 6.37 & 66.84 & 15.18 & 5.09 & 3.85 & 1.88 & 1.00 & 0.10 & 32.05 & 0.03 & 104.15 & 15.82 & 68.90 & 61.69 & 10.21 & 8.35 \\
\hline CROATIA & 2.10 & 6.67 & 69.97 & 15.89 & 5.33 & 4.03 & 1.97 & 1.04 & 0.11 & 33.55 & 0.03 & 109.03 & 16.56 & 72.13 & 64.58 & 10.69 & 8.74 \\
\hline
\end{tabular}




\section{SAŽETAK}

\section{Emisije iz sustava sakupljanja komunalnog otpada u Republici Hrvatskoj za 2013. godinu: Mješoviti otpad \\ Anamarija Grbeš, , $^{*}$ llijana Ljubićb i Želimir Veinovića}

U Republici Hrvatskoj odgovornost za uklanjanje otpada (sakupljanje i odlaganje) nose jedinice lokalne samouprave 21 županije (odnosno 20 županija i posebna jedinica Grad Zagreb). Uklanjanje otpada kao ekonomska aktivnost povjerena je tvrtkama sakupljačima otpada kojih je u Hrvatskoj u analiziranom razdoblju bilo 208, a organiziranim sakupljanjem je bilo obuhvaćeno 99 \% stanovništva. Sakupljanje mješovitog otpada iz kućanstava i poslovnih subjekata provodilo se različitom dinamikom, a otpad se iz kanti i spremnika preuzimao u kamione za otpad te prevozio do odlagališta odnosno postrojenja za oporabu.

U ovom radu procjenjuje se potrošnja dizelskog goriva te s njegovim izgaranjem povezane emisije u zrak uslijed sakupljanja mješovitog komunalnog otpada u Republici Hrvatskoj u 2013. godini. Ulazni podatci i rezultati prikazuju se za Hrvatsku te za svaku županiju posebno, u ukupnom iznosu te u relativnom iznosu (po stanovniku odnosno po jedinici mase prikupljenog otpada). Godišnja potrošnja dizelskog goriva u 2013. godini za sakupljanje mješovitog otpada procijenjena je na 10,6 milijuna litara. Na razini županija potrošnja je varirala od 87 tisuća do 2,2 milijuna litara (prosječno 504 tisuće litara po županiji). Ukupna emisija $\mathrm{CO}_{2}$ procijenjena je na 28000 tona, što na županijskoj razini varira od 231 do 5711 tona. Relativna emisija ugljikova dioksida varira od 3,3 do $13 \mathrm{~kg} \mathrm{CO}_{2}$ po stanovniku (prosječno 6,6 kg po stanovniku), odnosno 8,6-28,1 kg po toni prikupljenog otpada (prosječno $17 \mathrm{~kg} \mathrm{CO}$ po toni otpada). Prosječne vrijednosti emisije $\mathrm{CO}_{2}$ uslijed sakupljanja otpada, koje bi također trebale biti i ciljane vrijednosti optimizacije sakupljanja otpada, kreću se od 7 do 9 kg za mješoviti otpad te od 8 do 15 kg CO za različite, odvojeno prikupljene vrste otpada. Uz emisiju ugljikova dioksida, u ovom se radu prikazuju i emisije drugih tvari, kao što su $\mathrm{NO}_{x}, \mathrm{CO}, \mathrm{CO}_{2}$ od izgaranja maziva, NMVOC, $\mathrm{PM}, \mathrm{f}-\mathrm{BC}, \mathrm{N}_{2} \mathrm{O}, \mathrm{SO}_{2}, \mathrm{NH}_{3}, \mathrm{~Pb}$, ID[1,2,3-cd]P, B[k]F, B[b]F, B[a]P), te ukupna duljina transporta.

\section{Ključne riječi}

Sakupljanje komunalnog otpada, mješoviti otpad,

gospodarenje otpadom, potrošnja goriva, emisije u zrak

a Sveučilište u Zagrebu

Rudarsko-geološko-naftni fakultet

Stručni rad

${ }^{\mathrm{b}}$ Humana Nova Zagreb

Prispjelo 26. ožujka 2017. Prihvaćeno 14. rujna 2017. 